\title{
All Loop Topological String Amplitudes from Chern-Simons Theory
}

\section{Citation}

Aganagic, Mina, Marcos Mariño, and Cumrun Vafa. 2004. "All Loop Topological String Amplitudes from Chern-Simons Theory." Communications in Mathematical Physics 247 (2): 467-512. https:// doi.org/10.1007/s00220-004-1067-x.

\section{Permanent link}

http://nrs.harvard.edu/urn-3:HUL.InstRepos:41384996

\section{Terms of Use}

This article was downloaded from Harvard University's DASH repository, and is made available under the terms and conditions applicable to Other Posted Material, as set forth at http:// nrs.harvard.edu/urn-3:HUL.InstRepos:dash.current.terms-of-use\#LAA

\section{Share Your Story}

The Harvard community has made this article openly available.

Please share how this access benefits you. Submit a story.

Accessibility 
HUTP-02/A024

hep-th/0206164

\title{
All Loop Topological String Amplitudes From Chern-Simons Theory
}

\author{
Mina Aganagic, Marcos Mariño and Cumrun Vafa \\ Jefferson Physical Laboratory \\ Harvard University \\ Cambridge, MA 02138, USA
}

\begin{abstract}
We demonstrate the equivalence of all loop closed topological string amplitudes on toric local Calabi-Yau threefolds with computations of certain knot invariants for ChernSimons theory. We use this equivalence to compute the topological string amplitudes in certain cases to very high degree and to all genera. In particular we explicitly compute the topological string amplitudes for $\mathbb{P}^{2}$ up to degree 12 and $\mathbb{P}^{1} \times \mathbb{P}^{1}$ up to total degree 10 to all genera. This also leads to certain novel large $N$ dualities in the context of ordinary superstrings, involving duals of type II superstrings on local Calabi-Yau three-folds without any fluxes.
\end{abstract}

June 2002 


\section{Introduction}

In [1] it was conjectured that $U(N)$ Chern-Simons theory on $\mathbf{S}^{3}$, which describes topological A-model of $N$ D-branes on $X=T^{*} \mathbf{S}^{3}$, is dual at large $N$ to topological closed string theory on $X^{t}=\mathcal{O}(-1) \oplus \mathcal{O}(-1) \rightarrow \mathbb{P}^{1}$. There it was shown that the 't Hooft expansion of Chern-Simons free energy agrees with topological string amplitudes on $X^{t}$ to all genera. The conjecture was further tested in [2], where computations of certain Wilson loop observables in Chern-Simons theory were shown to match the corresponding quantities on $X^{t}$. Various aspects of the duality were studied in [3, 4, 5, 6, 7, 8, 9, 10] from different points of view. The topological string duality was embedded in the superstring theory in [11]. In [12] the target space derivation of the superstring duality of [11] was found by lifting up to M-theory [12, 13]. This was further studied in [14, [15], and also in a related context in [16, 17, 18, 19, 20,21,22,23]. Recently, [24] gave a world-sheet proof of the topological string duality based on some earlier ideas in [1].

In [25] a large class of new large $N$ dualities was proposed which generalize the conjecture of [1] to more general backgrounds, employing the philosophy of [1] that the large $N$ dualities are geometric transitions. On the open string side, replacing $T^{*} \mathbf{S}^{3}$ with a more general Calabi-Yau manifold $X$ led one to incorporate large open string instantons whose contributions deform Chern-Simons theory [26]. In the spirit of 't Hooft's original large $N$ conjecture, the holes in open string Riemann surfaces fill up at large $N$, and the complicated open string instanton sums that arise in a general Calabi-Yau $X$ get related to a complicated structure of instantons on the dual closed string side. Some important aspects of how this works were clarified in [27]. For one of the examples of [25], where both sides of the duality are explicitly computable to all orders [27] verifies the correspondence at the level of the partition functions. However, in a general setting, the descriptions of the theory in terms of open and closed strings are at the same level of complexity, and the duality was not easy to check (beyond the leading disk amplitude).

In this paper, by combining all of the ideas above together with several new technical ingredients, we show that Chern-Simons theory with product gauge groups and topological matter in bifundamental representations computes all loop topological string amplitudes on non-compact toric Calabi-Yau manifolds. Namely, it is shown that open string duals of a certain class of local toric Calabi-Yau manifolds involve D-branes on chains of Lagrangian submanifolds that are coupled only via annuli. In terms of Chern-Simons theory this is related to computations of appropriate combinations of Wilson loop observables associated 
with knots that are the boundaries of the annuli. The duality is local in the sense that, as in [1], the three-manifolds wrapped by D-branes get replaced by $\mathbb{P}^{1}$ 's in the dual. However in this case, open string theories build very complicated closed string geometries: in fact any noncompact toric Calabi-Yau manifold arises in some limit of this.

The paper is organized as follows. In section 2 we review the relevant geometries for open and closed strings that are related by large $N$ duality. In section 3 , we discuss the physics of open string theories, and explain why the model simplifies dramatically using a deformation argument. In section 4 we explain what is the relevant Chern-Simons computation in terms of three-manifolds glued with annuli. In section 5 we propose the large $N$ dualities and we argue that the results of [24] should be applicable to derive them. In section 6 we discuss the relation between the predictions of this duality to localization in the A-model closed string computation. In section 7 we present explicit evaluations of the amplitudes and provide predictions for the integer invariants for some examples including $\mathbb{P}^{2}$ and $\mathbb{P}^{1} \times \mathbb{P}^{1}$, and we show that they agree with the known results when they are available [28] [29] [30] [31]. In section 8 we consider embedding of this in the superstring context. Results of previous sections give open string duals of closed string geometries with no RR flux. Moreover, we show that some local geometries in IIB string theory have dual description in terms of gauge theory alone.

The work in section 7.2. was done in collaboration with P. Ramadevi, to whom we are very grateful. Also, our work has some overlap with the work of [32], and we thank the authors for discussing their work prior to publication. In particular we learned of their result that only a limited number of holomorphic curves contributes to the amplitudes before we found the general argument presented in section 3. The argument discussed in [32] (in the context of $d P_{2}$ ) uses localization principle, whereas our argument that only annuli contribute for all toric 3 -folds is based on complex structure deformation invariance.

\section{Geometry}

\subsection{Open String Geometry: $T^{2}$ Fibrations and Their Degenerations}

In this paper, the relevant Calabi-Yau manifolds are non-compact and admit a description as a special Lagrangian $T^{2} \times \mathbb{R}$ fibration over $\mathbb{R}^{3}$. The $T^{2}$ fibers degenerate over loci in the base. The geometry of the manifold is encoded in the one dimensional graphs 
in $\mathbb{R}^{3}$ that correspond to the discriminant of the fibration. A very familiar example of a Calabi-Yau manifold of this type is $X=T^{*} \mathbf{S}^{3}$. The complex structure of $X$ is given by

$$
x y=z, \quad u v=z+\mu,
$$

The two-torus is visible in the above equation as it is generated by two $U(1)$ isometries of $X$ acting as

$$
x, y, u, v \rightarrow x e^{i \alpha}, y e^{-i \alpha}, u e^{i \beta}, v e^{-i \beta} .
$$

The $\alpha$ and $\beta$ actions above can be taken to generate the $(1,0)$ and $(0,1)$ cycle of the $T^{2}$.

The local type of the singularity has a $T^{2}$ fiber that degenerates to $\mathbf{S}^{1}$ by collapsing one of its one-cycles. In the equation above, the $U(1)_{\alpha}$ action fixes $x=0=y$ and therefore fails to generate a circle there. In the total space, the locus where this happens, i.e. the $x=0=y=z$ subspace of $X$, is another cylinder $u v=\mu$. The projection to the base space forgets the circle of this cylinder and is a line in $\mathbb{R}^{3}$.

Such a geometry locally looks like a Taub-Nut (TN) space times a cylinder $\mathbf{C}^{*}=$ $\mathbb{R} \times \mathbf{S}^{1}$. Here, the TN space itself is thought of as a cylinder $x y=$ const which is fibered over the $z$ plane and which degenerates at $z=0$. Analogous considerations apply to the $U(1)_{\beta}$ action. The locus of degenerate fibers in the base $\mathbb{R}^{3}$ of the deformed conifold is given in the figure below. In this and similar figures below, two of the directions of the base are the axes of the two cylinders, and the third direction represents the real axis of the $z$-plane.

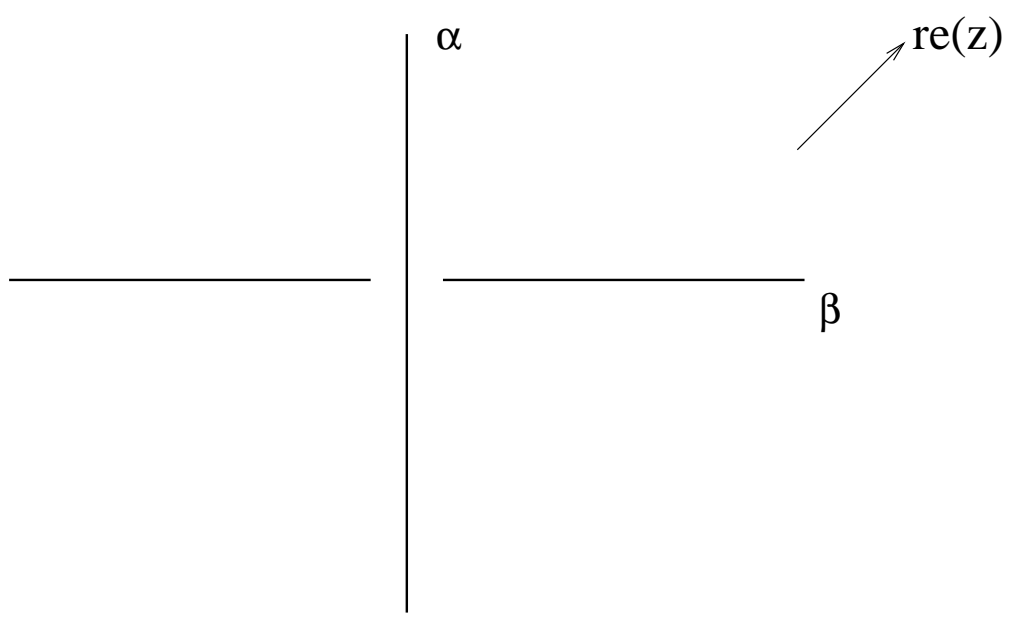

Fig. 1 The figure depicts the discriminant locus of the $T^{2} \times \mathbb{R}$ fibration in the base $\mathbb{R}^{3}$. The $\alpha$ and $\beta$ cycles of the $T^{2}$ degenerate over lines $z=0, z=-\mu$. 
In general, any $(p, q)$ cycle of the $T^{2}$ can degenerate in this way. As long as the degenerate loci do not intersect, the local geometry is that of Taub-Nut space, as an $\operatorname{SL}(2, \mathbf{Z})$ transformation on the $T^{2}$ fiber can be used to relate it to the degenerations discussed above. In what follows, it will be important that the orientation of the locus where the $T^{2}$ fiber degenerates in the base $\mathbb{R}^{3}$ is correlated with the $(p, q)$ type of degenerating cycle. We have seen an example of this above in the case of $T^{*} \mathbf{S}^{3}$, where the $\alpha$ and $\beta$ cycles degenerated along orthogonal directions in the base in fig. 1. The origin of this is the fact that the Calabi-Yau manifold is a complex manifold and the fibration is special Lagrangian.

We will not go into detail here in this language as it is cumbersome for physicists, and explained in the literature (see for example 250) 1 , especially because there is a string theory duality that provides excellent intuition about the geometry, which we would like to explain instead.

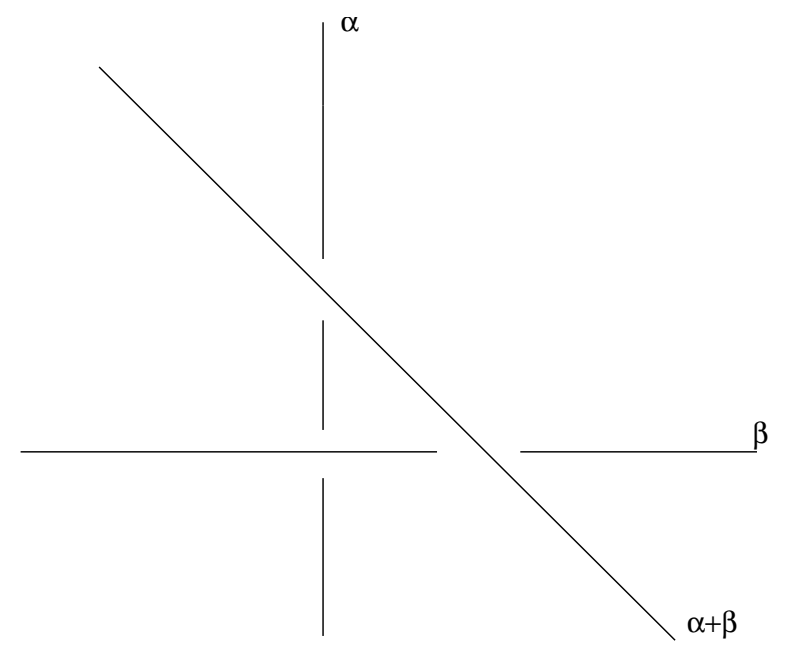

Fig. 2 The degeneration locus of the $T^{2}$ fibration in the base specifies the Calabi-Yau geometry. The orientation of the lines are related to the $(p, q)$ type of the 1-cycle that degenerates over it. In the type IIB language, this corresponds to different $(p, q)$ fivebranes.

1 To give an idea of the more general situation, let $\hat{x}_{\alpha, \beta}$ be the single valued holomorphic coordinates on $\mathbf{C}^{*} \times \mathbf{C}^{*}$, and let $z$ be a coordinate on $\mathbb{R}^{2}$. If a $(p, q)$ cycle of the $T^{2}$ degenerates at a point in $z$, then the fixed point locus which is invariant under $\hat{x}_{\alpha} \rightarrow \hat{x}_{\alpha} \mathrm{e}^{i p \theta}, \hat{x}_{\beta} \rightarrow \hat{x}_{\beta} \mathrm{e}^{i q \theta}$. In terms of periodic variables $\hat{x}_{\alpha, \beta}=\exp \left(x_{\alpha, \beta}\right)$ we can write the degeneration locus as by

$$
q x_{\alpha}-p x_{\beta}=\text { const. }
$$




\subsection{Relation to $(p, q)$ Fivebranes in IIB}

In this section we connect the description of Calabi-Yau geometry by a duality to the web of $(p, q)$ fivebranes [33]. This will be helpful for us for an intuitive picture of holomorphic curves in the geometry. The connection was derived in [34] and we will now review it.

Recall that M-theory on $T^{2}$ is related to type IIB string theory on $\mathbf{S}^{1}$. Since the Calabi-Yau manifolds we have been considering are $T^{2}$ fibered over $B=\mathbb{R}^{4}$, we can relate geometric M theory compactification on Calabi-Yau manifold $X$ to type IIB on flat space on $B \times \mathbf{S}^{1}$. However, due to the fact that $T^{2}$ is not fibered trivially, this is not related to the vacuum type IIB compactification.

The local type of singularity, as we have seen above, is the Taub Nut space $T N_{p, q}$, where the $(p, q)$ label denotes which cycle of the $T^{2}$ corresponds to the $\mathbf{S}^{1}$ of the Taub-Nut geometry. Under the duality, this local degeneration of $X$ is mapped to the $(p, q)$ fivebrane that wraps the discriminant locus in the base space $B$, and lives on a point on the $\mathbf{S}^{1}$. The fact that the $(p, q)$ type of the five brane is correlated with its orientation in the base is a consequence of the BPS condition. More precisely, configurations of five branes that preserve supersymmetry and $4+1$-dimensional Lorentz invariance are pointlike in a

fixed $\mathbb{R}^{2}$ subspace of the base that we called the $z$ plane above. In the two remaining directions of the base, the five branes are lines where the equation of the $(p, q)$ five brane is $p x_{\alpha}+q x_{\beta}=$ const.

\subsection{Geometric Transitions}

Consider a pair of lines in the base space over which two one-cycles of the $T^{2}$ degenerate. Any path in the base space ending on the two lines, together with the $T^{2}$ fiber over it, gives rise to a closed three-manifold in the total space. This is because a cycle of the $T^{2}$ degenerates over the start and the end point of the path, so the three-manifold has no boundaries. If the two lines intersect in the base space, the three-cycle obtained in this way can be shrunken to a point. If they don't, it generates a homology class in $H_{3}(X, \mathbf{Z})$. Let $n$ be the number of five-branes. If the five branes are in generic positions and do not intersect, the manifold is smooth, and it is easy to see that the the dimension of third homology is $b_{3}(X)=n-1$.

In the superstring context, among Lagrangian three-cycles in the Calabi-Yau manifold, special Lagrangian three-cycles are of particular interest as they are supersymmetric, i.e. 
D-branes wrapped on them preserve some supersymmetry of the theory. These cycles are volume-minimizing and project to paths of shortest length in the base (the Lagrangian condition can always be satisfied with some choice of symplectic form on $X$ ). In the non-compact situation we are discussing, the meaning of this is particularly transparent in the IIB string theory as the five-branes live in $\mathbb{R}^{4}$ with flat metric. The number of supersymmetric cycles, for five-branes in generic positions, is easily counted by doing the projection of the base $\mathbb{R}^{3} \rightarrow \mathbb{R}^{2}$ that suppresses the $z$-direction and counts the number of intersections. Generically there will be $n(n-1) / 2$ such intersection points (unless some $(p, q)$ 5-branes are of the same type).

The Calabi-Yau manifolds we have been discussing have geometric transitions where three-cycles in geometry shrink and the resulting singularity is smoothed to a manifold $X^{t}$ of different topology. This was explained in some detail in [25]. In the examples we will be studying in this paper, the local geometry of the singularity will be $T^{*} \mathbf{S}^{3}$, so the geometric transition in question involves an $\mathbf{S}^{3}$ shrinking and a $\mathbb{P}^{1}$ growing. The geometric transitions do not spoil the fact that the manifold is $T^{2}$ fibered, however they do change the locus of singular fibers. After the transition that shrinks all the three-cycles (and these always exist in the family of $X$ 's we consider), the resulting manifolds are toric varieties. Toric varieties admit a group of $U(1)$ isometries whose rank is the complex dimension of the manifold. In our case this is $U(1)^{3}$, and the symmetry enhancement comes from the fact that the transition which gets rid of all the three-cycles requires all the loci of singular fibers to coincide in the $z$-plane, and the extra $U(1)$ is the group of rotations about this point. While the reader might get an impression from the above discussion that the manifold after transition gains new cycles only in $H_{2}\left(X^{t}, \mathbf{Z}\right)$, this is in fact not the case. In fact, in the generic case the number of shrinking minimal three-cycles is larger than the number of classes in $H_{3}(X)$. Then, since not all three-cycles are independent in homology, there are four-chains with boundaries on some of them corresponding to the relations which they satisfy. After the transition, the four-chains close off because their boundaries shrink. As a consequence, the dual geometry does involve compact cycles in $H_{4}(X, \mathbf{Z})$. 

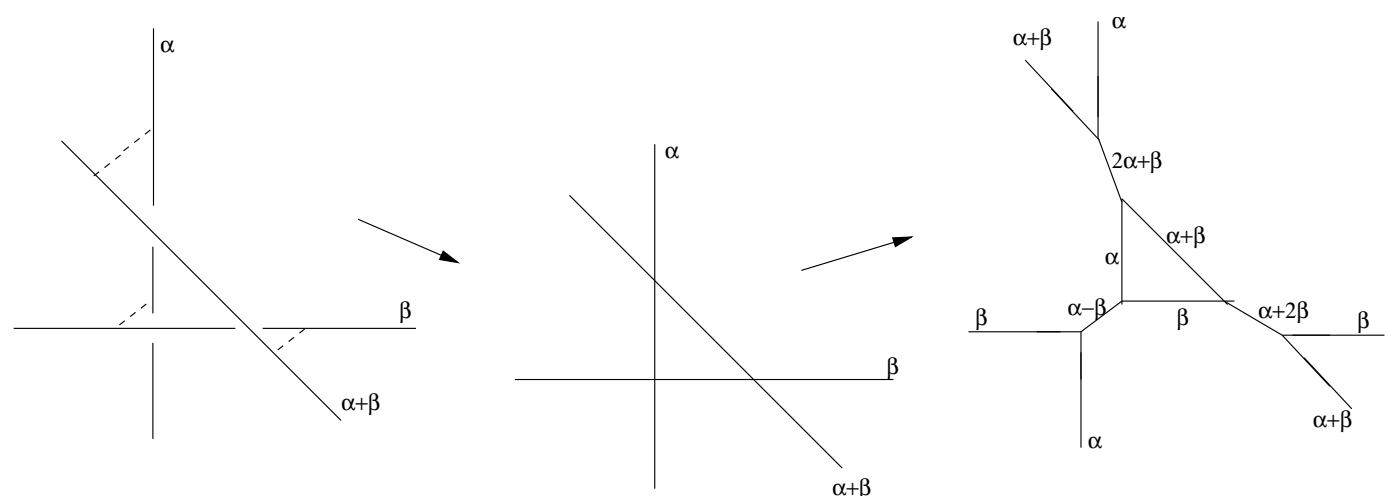

Fig. 3 This shows the geometric transition of the Calabi-Yau in the previous figure. In the leftmost geometry there are three minimal 3-cycles. The lengths of the dashed lines are proportional to their sizes. The intermediate geometry is singular, and the figure on the right is the base of the smooth toric Calabi-Yau after the transition. This Calabi-Yau is related to $\mathbb{B}_{3}$ by flopping three $\mathbb{P}^{1}$ 's.

In the language of $(p, q)$ five branes, the geometric transition corresponds to a phase transition in the five-dimensional theory. Namely, the configuration of intersecting $(1,0)$ and $(0,1)$ five-branes is a phase transition point: the Higgs phase with five-branes separated in the $z$ plane 2 meets the Coulomb phase, where a piece of $(1,1)$ brane resolves the singularity. In the geometry, there is a $T^{2}$ fiber whose $(1,1)$ cycle degenerates over this interval, and the cylinder is capped off to a $\mathbb{P}^{1}$ by all the cycles of the $T^{2}$ degenerating over the boundaries of the interval. The singularity can also be resolved with a $(1,-1)$ brane, which corresponds to the flopped $\mathbb{P}^{1}$.

\subsection{Geometry of Holomorphic Curves}

Calabi-Yau manifolds generally come with families of embedded curves. In the topological A-model only holomorphic curves are relevant, as the A-model string amplitudes localize on them. In the presence of D-branes wrapping Lagrangian submanifolds $M_{i}$ in $X$, we must also consider holomorphic curves with boundaries on the $M_{i}$ 's.

Holomorphic curves have a very simple description in the toric base, or equivalently, in the $(p, q)$ five brane language. Let us first consider closed string geometries, the family

2 Recall that this is the complex structure modulus of the geometry. The five dimensional hyper-multiplet contains a compact scalar from the period of the $C$-field through the $\mathbf{S}^{3}$ (or the positions of the five-branes on the $\mathbf{S}^{1}$ in type IIB). It also has a non-compact scalar from the "period" of the $C$-field through the non-compact three-cycle dual to $\mathbf{S}^{3}$ or the "Wilson line on $\mathbb{R}$ " for the five-brane. Topology-changing transitions of toric manifolds are discussed in [35]. 
of Calabi-Yau manifolds we have called $X^{t}$ above. In this case, it can be shown that all the compact holomorphic curves in a non-compact toric Calabi-Yau manifold wrap a 1-cycle in the $T^{2}$ fiber direction. Holomorphic curves project to lines in the toric base, and locally the direction of the curve in the base is correlated with its direction in the fiber. For the compact curves, the direction in the fiber is the $(p, q)$ 1-cycle of the $T^{2}$. This is most transparent in the $(p, q)$ five-brane language.
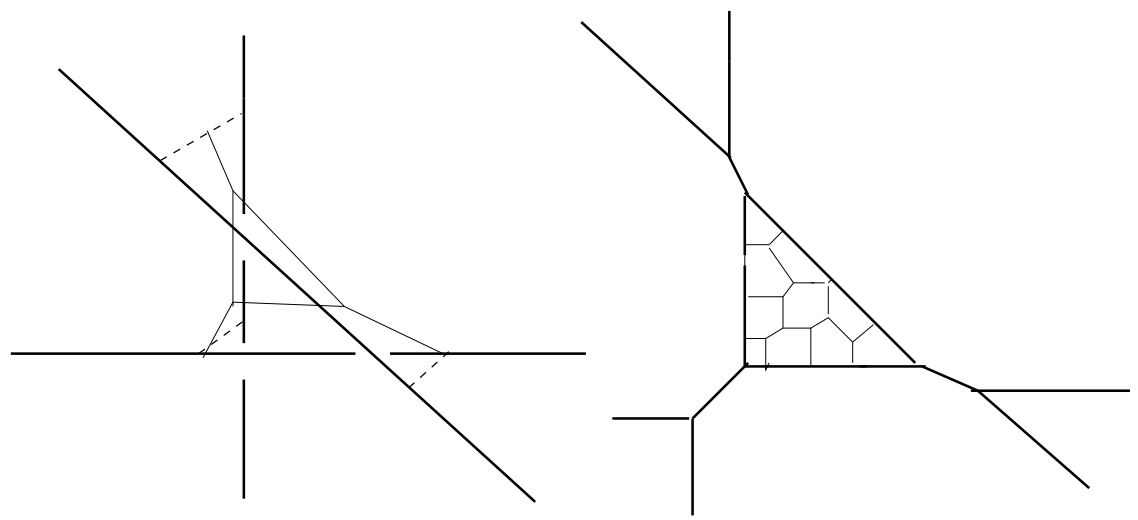

Fig. 4 The figure on the left depicts a genus one holomorphic curve with three holes ending on three minimal three-cycles. The figure on the right is after the transition, and also depicts a genus one curve, but without boundaries.

Namely, consider an M-theory membrane, wrapping a holomorphic curve on $X^{t}$. By M-theory/type IIB duality, a membrane wrapping a $(p, q)$ cycle of the $T^{2}$ is dual to a $(p, q)$ string, therefore membranes on holomorphic curves in $X^{t}$ that are along the $T^{2}$ in the fiber are dual to webs of $(p, q)$ strings in type IIB string theory that are BPS. Moreover, the compact curves are related to webs ending on $(p, q)$ five-branes [33]. An example of such a curve is given in the right portion of fig. 4 .

Much of the same considerations are clearly true in $X$ as well. There is however an important distinction from the point of view of topological string amplitudes. Namely, in a generic situation, there are no compact holomorphic curves. This is easy to see in the $(p, q)$ five-brane picture because a condition for the web to be supersymmetric is that it lives in the plane parallel to the web of the five branes, and is therefore pointlike in the $z$ plane (there is an additional condition that fixes the orientation of the $(p, q)$ string depending on its charge, which comes from the balance of tensions, by requiring it to be orthogonal to a $(p, q)$ five brane. We refer the reader to [33] for a detailed discussion). However, a given $(p, q)$ string can only end on the five brane of the same charge, and so for five-branes at 
generic locations in the $z$ plane, the string webs are never compact. This situation changes if strings can end elsewhere. For example, if there are M-theory five-branes wrapped on Lagrangian cycles in $X$, membranes can end on them. The M-theory five-brane is replaced with a D3-brane in IIB string theory ending on the various $(p, q)$ five-branes. There are then compact string webs ending on the D3-branes, corresponding to holomorphic curves with boundaries. In relation to large $N$ dualities in superstring context it is more natural to consider type IIA string on $X$ instead, with D6-branes on the $M_{i}$ 's. This is related, via duality of IIA/M-theory on $\mathbf{S}^{1}$, to IIB string theory with Kaluza-Klein monopoles ending on the $(p, q)$ five brane web [7]. Namely, the D6 branes in Lagrangian submanifolds of $X$ lift to M-theory on a $G_{2}$ holonomy manifold. To obtain this manifold, we need to consider an extra $\mathbf{S}^{1}$ which is fibered over the corresponding CY. This is related to IIB on $B \times \mathbf{S}^{1}$ where we exchange the 11-th circle with the $T^{2}$ that fibers $X$. What used to be the 11-th circle is now fibered nontrivially over $B$. In particular, the circle vanishes over a 2-dimensional subspace of the type IIB 5-dimensional geometry. It vanishes along the line in $B$ ending on the $(p, q)$ five branes as well as on the $\mathbf{S}^{1}$ which is dual to the $T^{2}$ of M-theory. This line in $B$ corresponds to the line in the base of $X$ over which there is the three-manifold that the D6 brane wraps. The IIB 5-brane web is in a background of ALF geometry dictated by the location of the Lagrangian submanifold in $X$.

\subsection{Geometry of Three-Cycles}

In this paper, we will wrap D-branes on the minimal three-manifolds in $X$. The physics of the D-branes depends on both $X$ and the three-manifold $M$ it is wrapped on, so we will describe the geometries of the latter.

In our context, $M$ is obtained by pinching the cycles of the $T^{2}$ fibers over the endpoints of an interval in the base. Clearly, if the same cycle vanishes at both ends, the topology of the three-manifold is $\mathbf{S}^{2} \times \mathbf{S}^{1}$, as there is a cycle of the $T^{2}$ that never vanishes on $M$. An example where the manifold is $\mathbf{S}^{3}$ arises in the familiar context of $T^{*} \mathbf{S}^{3}$. This $\mathbf{S}^{3}$ comes from a $(1,0)$ cycle of the $T^{2}$ vanishing at one end, and $(0,1)$ cycle vanishing on the other. To see that this is an $\mathbf{S}^{3}$, note that at $x=\bar{y}$ and $u=-\bar{v}$ the equation (2.1) defining $T^{*} \mathbf{S}^{3}$ becomes

$$
|x|^{2}+|u|^{2}=\mu,
$$

and $\mu$ is real and positive, so this is a three-sphere. In view of the discussion above, we can regard this as a real interval, together with the $(1,0)$ one-cycle that corresponds to 
the phase of $x=\bar{y}$, degenerating at the end with $x=0$, and the $(0,1)$ cycle that is the phase of $u=-\bar{v}$ degenerating over the $u=0$ endpoint 3 .

More generally, we have the following. For our current purpose, by an $\mathrm{SL}(2, \mathbf{Z})$ transformation of the $T^{2}$ we can make $(1,0)$ be the vanishing cycle over one of the boundaries, and let $(q, p)$ be the cycle that vanish over the other. The 3-manifold itself is a Lens space $L(p, q)$. Remember that lens spaces are defined as quotients of $\mathbf{S}^{3}$ by a $\mathbf{Z}_{p}$ action. The space $L(p, q)$ is given by

$$
|x|^{2}+|u|^{2}=1 \quad(x, u) \sim(\exp (2 i \pi / p) x, \exp (2 i \pi q / p) u)
$$

To see that, consider an $\mathbf{S}^{3}$ which, as explained above, is a $T^{2}$ fibration over an interval, where the cycles of the $T^{2}$ are generated by phases of $x, u$. If the complex structure of the $T^{2}$ corresponding to $\mathbf{S}^{3}$ it is $\tau$, then an $\operatorname{SL}(2, \mathbf{Z})$ transformation that takes this $T^{2}$ to a $T^{2}$ with $(1,0)$ and $(q, p)$ cycles vanishing over the endpoints will take $\tau$ to $\tau^{\prime}=\frac{\tau+q}{p}$. But the $T^{2}$ with the new complex structure is precisely a quotient of the original one by the $\mathbf{Z}_{p}$ action specified in $(2.2)$. Note that $L(p, q)$ is homeomorphic to $L(p, 1)$. In the present context this corresponds to the fact that global $\mathrm{SL}(2, \mathbf{Z})$ transformations preserving the $(1,0)$ cycle of the $T^{2}$ can be used to set $q$ to one.

For our later considerations in this paper it is important to have another view on this construction of a three-manifold $M$ as a $T^{2}$ fiber over interval. The construction is as follows: we are gluing two solid tori over (say) the midpoint of the interval, up to an $\mathrm{SL}(2, \mathbf{Z})$ transformation $V_{M}$ that corresponds to a diffeomorphism identification of their boundaries. Let us call the two tori on each side of the midpoint by $T_{L}^{2}$ and $T_{R}^{2}$. The embedding of this in the Calabi-Yau geometry provides a canonical choice of $V_{M}$. In the Calabi-Yau geometry, there is a natural choice of basis of cycles $\alpha, \beta$ of the $T^{2}$ that fibers $X$, which is provided by the choice of complex structure on $X$. We can identify the one-cycles of the $T^{2}$ fiber that shrink over the left and the right sides of the interval with the shrinking 1-cycles of $T_{L}$ and $T_{R}$. The diffeomorphism map $V_{M}$ is the $\operatorname{SL}(2, \mathbf{Z})$ transformation that relates one of the shrinking cycles of the fiber of $X$ to the other one.

Let us now explain the construction of the gluing matrices that will suit our purpose. Let $\left(p_{L}, q_{L}\right)$ be the cycle of the $T^{2}$ fiber that degenerates over the left half on $M$, and let

3 This is in fact the minimal $\mathbf{S}^{3}$ in $X$, as it is a fixed point set of the real involution on (2.1) given by $x \rightarrow \bar{y}$ and $u \rightarrow-\bar{v}$. 
$\left(p_{R}, q_{R}\right)$ be the cycle that degenerates over the right half. The gluing matrix $V_{M}$ can be written as

$$
V_{M}=V_{L}^{-1} V_{R}
$$

where $V_{L, R}=\left(\begin{array}{cc}p_{L, R} & s_{L, R} \\ q_{L, R} & t_{L, R}\end{array}\right) \in S L(2, \mathbf{Z})$ Clearly, $V_{M}$ is unique up to a homeomorphism that changes the "framing" of three-manifold [36] and takes

$$
V_{L, R} \rightarrow V_{L, R} T^{n_{L, R}}
$$

where $T$ is a generator of $S L(2, \mathbf{Z}), T=\left(\begin{array}{ll}1 & 1 \\ 0 & 1\end{array}\right)$. This is a consequence of the fact that there is no natural choice of the cycle that is finite on the solid torus.

In the case of $M=\mathbf{S}^{3}$ above, since $(1,0)$ degenerates in the left half of $M$ and $(0,1)$ in the right half, $V_{M}=S$, where $S=\left(\begin{array}{cc}0 & -1 \\ 1 & 0\end{array}\right)$. As a small modification, we could make $(p, 1)$ degenerate over the left half instead, so that $V_{L}=T^{p} S$ is a lens space $L(p, 1)$ and $V$ is $S^{-1} T^{-p} S$. For most considerations in this paper we will be considering the cases $L(1,1)$ or $L(1,0)$, which are homeomorphic to $\mathbf{S}^{3}$.

\section{Open String Theory}

We are interested in the topological A-model on the Calabi-Yau geometries described above, with D-branes wrapping special Lagrangian three-spheres. The local geometry in some neighborhood of a Lagrangian three-manifold $M$ is $T^{*} M$ and it was shown in [26] that the topological A-model corresponding to $N$ D-branes on $M$ is a $U(N)$ Chern-Simons theory on three-manifold $M$,

$$
Z=\int \mathcal{D} A e^{S_{\mathrm{CS}}(A)}
$$

where

$$
S_{\mathrm{CS}}(A)=\frac{i k}{4 \pi} \int_{M} \operatorname{Tr}\left(A \wedge d A+\frac{2}{3} A \wedge A \wedge A\right)
$$

is the Chern-Simons action. The basic idea of this equivalence is as follows: the pathintegral of the topological A-model localizes on holomorphic curves. When there are Dbranes, this means holomorphic curves with boundaries ending on them. In the $T^{*} M$ geometry with D-branes wrapping $M$ there are no honest holomorphic curves, however there are degenerate holomorphic curves that look like trivalent ribbon graphs and come from the boundaries of the moduli space. This leads to a field theory description in target space, which is equivalent to topological Chern-Simons theory (as the abstract open string 
field theory formulation demonstrates [37]). In this map, the level $k$ would be naively related to the inverse of the string coupling constant $g_{s}$. However, quantum corrections [36] shift this identification to

$$
\frac{2 \pi i}{k+N}=g_{s} .
$$

More globally, however, the geometry is generally not that of the cotangent space to any manifold, and there can be D-branes wrapping other minimal three-spheres in $X$. In this case the topological open strings will have contributions from degenerate holomorphic curves, which are captured by Chern-Simons theories, as well as some honest holomorphic curves, which lead to insertion of some Wilson loop observables for the Chern-Simons theory [26]. If we have a number of $M_{i}$ 's distributed in some way inside a Calabi-Yau, with $N_{i}$ D-branes wrapped over $M_{i}$, then we can trade the degenerate holomorphic curves by including the corresponding Chern-Simons theories $S_{i}=S_{\mathrm{CS}}\left(A_{i}\right)$ coupled in an appropriate way with the honest holomorphic curves. Namely, we have

$$
e^{F_{\text {all }}}=\int \prod_{i} D A_{i} e^{S_{i}+F_{\text {ndg }}\left(U_{i}\left(\gamma_{i}\right)\right)}
$$

where $F_{\text {all }}$ denotes the full topological A-model amplitude, and $F_{\text {ndg }}$ denotes the contribution of the non-degenerate holomorphic curves to the topological amplitudes. These holomorphic curves give rise to Wilson loops on the D-branes: each holomorphic curve with area $A$ ending on $M_{i}$ over the knot $\gamma_{i}$ leads to the contribution $e^{-A} \prod_{i} \operatorname{Tr} U_{i}\left(\gamma_{i}\right)$ to $F_{\text {ndg }}$, where $U_{i}\left(\gamma_{i}\right)$ denotes the holonomy of the Chern-Simons gauge connection around the knot $\gamma_{i}$. Notice that all these Chern-Simons theories have the same coupling constant. More precisely,

$$
\frac{2 \pi i}{k_{i}+N_{i}}=g_{s}
$$

In the toric examples we will consider in this paper it turns out that only holomorphic annuli contribute to $F_{\text {ndg }}$ and thus this connection with Chern-Simons theory is a useful way to compute the topological A-model amplitudes as some particular correlation function in a system of coupled Chern-Simons theories. 


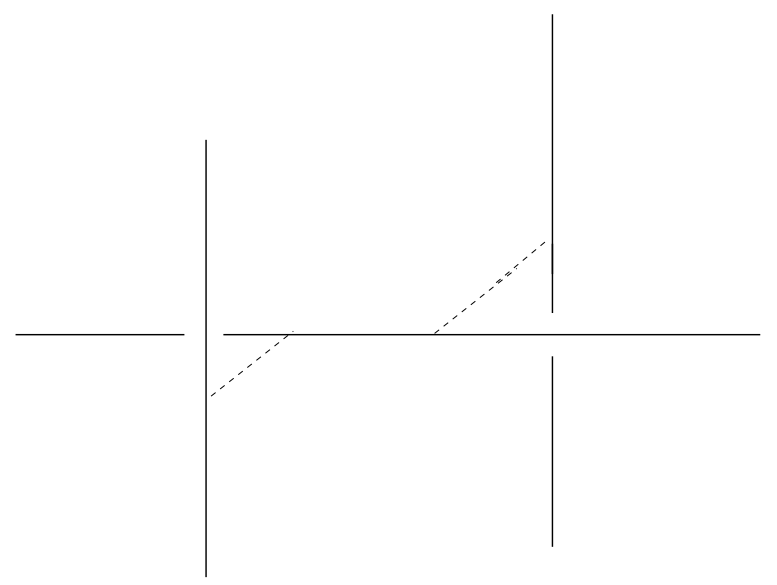

Fig. 5 Calabi-Yau geometry with $b_{2}=1, b_{3}=2$ and two minimal $\mathbf{S}^{3}$ 's as the dashed lines.

\subsection{The Annulus Amplitude}

As an example, let us consider the Calabi-Yau manifold $X$ with $b_{3}=2, b_{2}=1$ in fig. 5, whose complex structure is described by

$$
\begin{aligned}
x y & =z \\
x^{\prime} y^{\prime} & =\left(z-\mu_{1}\right)\left(z-\mu_{2}\right) .
\end{aligned}
$$

and was studied in a physics context in [38]. In $X$, the $\alpha$ cycle of the $T^{2}$ degenerates over the point $z=0$, but the $\beta$ cycle degenerates twice, over $z=\mu_{1}$ and $\mu_{2}$. The cycles over $\left[\mu_{1}, 0\right]$ and $\left[0, \mu_{2}\right]$ are three-spheres $M_{1,2}$ that generate $H_{3}(X)$. The base space of $X$, with loci with degenerate fibers - is pictured in fig. 5 , where we have taken $\mu$ 's to be real, and $\mu_{1}<0<\mu_{2}$ (the reader should keep in mind that only one of the dimensions of the $z$ plane is visible in the base).

As is clear from the picture, there is an additional parameter visible in the base: the relative distance of two $\beta$-branes. This is a Kähler parameter corresponding to the one compact 2-cycle in $X$ (since $X$ contains an $\mathbf{S}^{2} \times \mathbf{S}^{1}$, as we discussed above, it certainly contains an $\mathbf{S}^{2}$ that cannot be contracted - we simply pick a point on the $\mathbf{S}^{\mathbf{1}}$ in $\mathbf{S}^{2} \times \mathbf{S}^{1}$ ) 田.

4 The interpretation of this Kähler parameter is obvious in the type IIB dual, as it is the scalar field of the six dimensional $\mathcal{N}=(1,1)$ supersymmetric theory on the two parallel $(0,1)$ five-branes. Duality relates this to a Calabi-Yau three-fold in M-theory containing a curve of $A_{1}$ singularities [38]. 
As we discussed above, at the level of topological strings the theory is a $U\left(N_{1}\right) \times U\left(N_{2}\right)$ Chern-Simons theory, but since there are two stacks of D-branes, there is a new open string sector where one end of the string is on the D-branes wrapping $M_{1}$ and the other on $M_{2}$. The ground states of this string correspond to constant maps to the $\mathbf{S}^{1}$ that the threemanifolds "intersect" over. Correspondingly, there are two states in the Ramond sector of the topological string, a real scalar and a one form, with $U(1)_{R}$ charges $-1 / 2$ and $1 / 2$. Only the scalar is physical, and taking into account both orientations of the string, we get a complex scalar $\phi$ in $\left(N_{1}, \bar{N}_{2}\right)$. This complex scalar is generically massive, and its mass is proportional to the "distance" between $M_{1}$ and $M_{2}$ given by the complexified Kähler parameter $r$. We will show below that the only modification of the topological string we need to make in this geometry is to include the minimally coupled complex scalar in this sector. Because of the topological invariance of the theory the action of a charged scalar with minimal coupling is of the form $\mathcal{L}_{\phi} \sim \oint_{\gamma} \operatorname{Tr} \bar{\phi}\left(d-A_{1}+A_{2}\right) \phi$. Note that the scalar field gets a mass from turning on a Wilson line on the $\mathbf{S}^{1}$ it propagates on. We will pick its "background" value which we denote by $r$ below. The path integral involving $\phi$ is Gaussian so it can be easily evaluated [2] and gives:

$$
\begin{aligned}
\mathcal{O}\left(U_{1}, U_{2} ; r\right) & =\exp \left[-\operatorname{Tr} \log \left(\mathrm{e}^{r / 2} U_{1}^{-1 / 2} \otimes U_{2}^{1 / 2}-\mathrm{e}^{-r / 2} U_{1}^{1 / 2} \otimes U_{2}^{-1 / 2}\right)\right] \\
& =\exp \left\{\sum_{n=1}^{\infty} \frac{\mathrm{e}^{-n r}}{n} \operatorname{Tr} U_{1}^{n} \operatorname{Tr} U_{2}^{-n}\right\},
\end{aligned}
$$

where $U_{1,2}$ are the holonomies of the corresponding gauge fields around a loop 5

$$
U_{i}=\mathrm{P} \exp \oint_{\gamma} A_{i} \in U\left(N_{i}\right), \quad i=1,2 .
$$

5 In going from the first to the second line in (3.3), we have dropped a factor of $\operatorname{det}\left(U_{1}^{1 / 2}\right) \operatorname{det}\left(U_{2}^{-1 / 2}\right)$ in $\mathcal{O}$. This factor, which equals $\exp \left(\frac{\sqrt{N_{1}}}{2} \oint_{\gamma} \operatorname{Tr} A_{1}-\frac{\sqrt{N_{2}}}{2} \oint_{\gamma} \operatorname{Tr} A_{2}\right)$, can be absorbed away in a redefinition of $r$. It is likely that this is related to the holomorphic anomaly of topological strings [39], and this clearly deserves further investigation. 


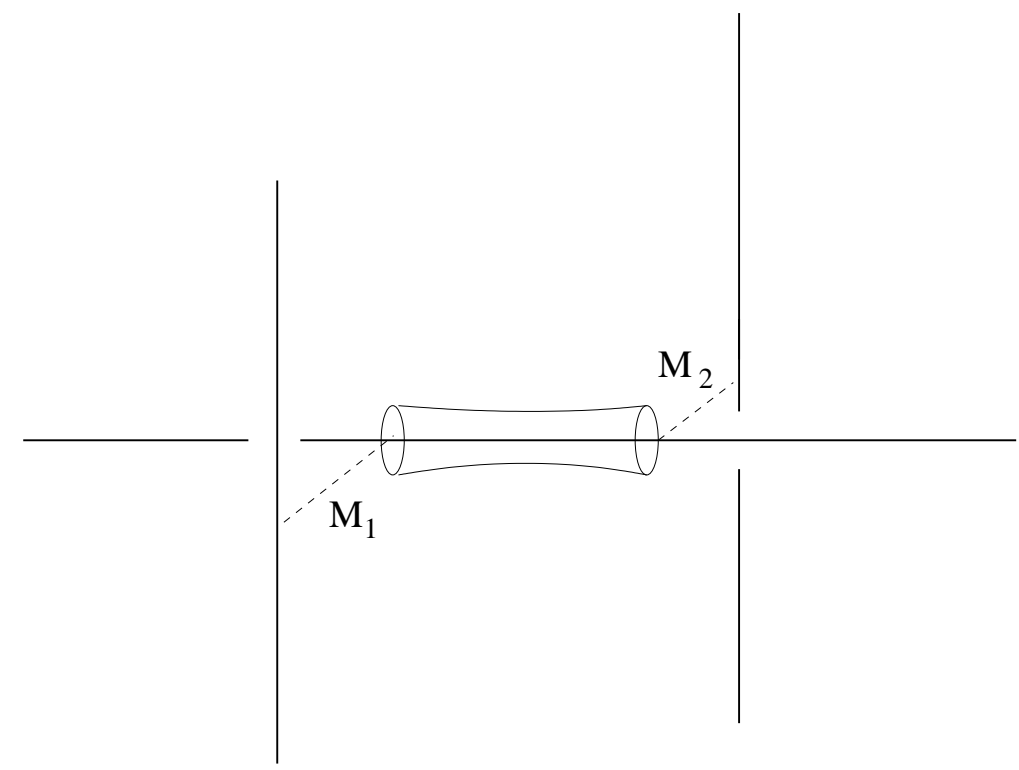

Fig. 6 There is one holomorphic annulus connecting the two $\mathbf{S}^{3}$ 's. This corresponds to a one-loop computation with a bifundamental string running around the loop.

Note that the operator $\mathcal{O}$ is the amplitude for a primitive annulus of size $r$ with boundaries on $M_{1}$ and $M_{2}$, together with its multicovers [2] [5] [8]. This annulus is depicted in fig. 6 , and it is a piece of the holomorphic curve that is wrapped by the $(1,0)$ brane. This curve is obtained by setting $x=0=y=z$ in (3.2) and is given by $x^{\prime} y^{\prime}=\mu_{1} \mu_{2}$.

The Chern-Simons path integral in this geometry is therefore defined with the insertion of the above operator. The path integral of the A-model string field theory in this background is therefore given by:

$$
Z=\int \mathcal{D} A_{1} \mathcal{D} A_{2} e^{S_{\mathrm{CS}}\left(A_{1}\right)+S_{\mathrm{CS}}\left(A_{2}\right)} \exp \left\{-\sum_{n=1}^{\infty} \frac{\mathrm{e}^{-n r}}{n} \operatorname{Tr} U_{1}^{n} \operatorname{Tr} U_{2}^{-n}\right\}
$$

To recapitulate, we have Chern Simons theory on two three-manifolds $M_{1}$ and $M_{2}$ connected via an annulus. The boundaries of the annulus look like $\mathbf{S}^{1}$ 's in both of them, i.e. we have one knot in each $M_{i}$. The topological theory is computing the expectation value of the operator $\mathcal{O}\left(U_{1}, U_{2} ; r\right)$, which involves Wilson loop operators around the two knots. This obviously extends to more general configurations: for every pair of three-manifolds $M_{1}$ and $M_{2}$ that are connected by a holomorphic annulus, we will get a bifundamental complex scalar. Integrating this field out, we find we need to insert an operator (3.3), where $r$ is the size of the corresponding annulus diagram in spacetime. 

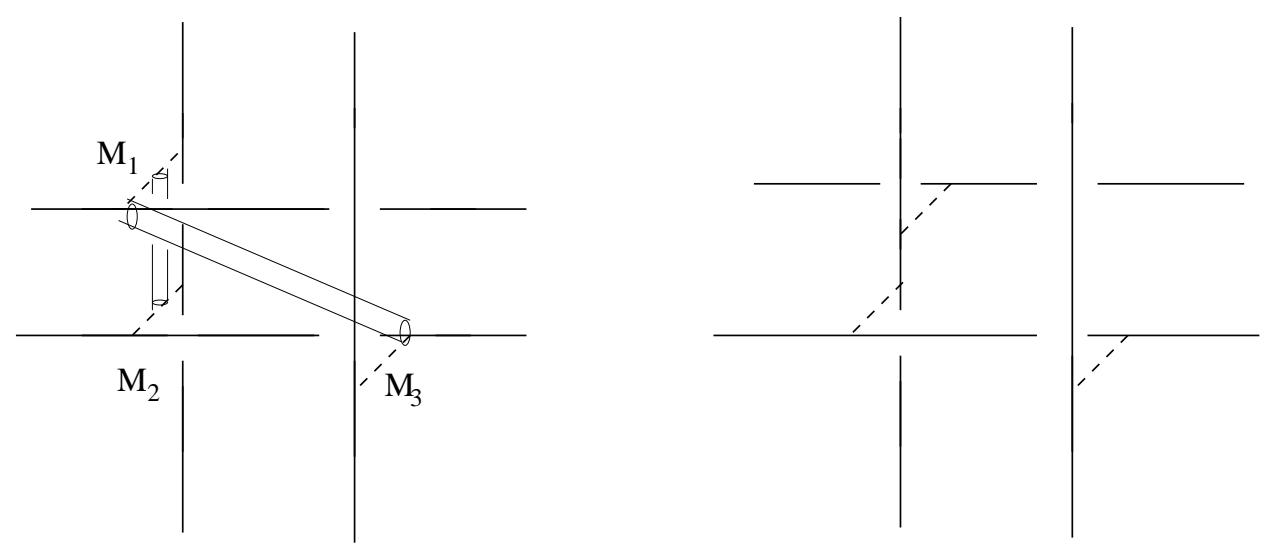

Fig. 7 In the left figure, it looks like there is a family of holomorphic annuli between $M_{1}$ and $M_{2}$, and a holomorphic annulus connecting $M_{1}$ with $M_{3}$. However, by moving in the complex structure moduli space we get to the figure in the right, where it is clear that there is an isolated holomorphic annulus connecting $M_{1}$ and $M_{2}$ and no holomorphic curve between $M_{1}$ and $M_{3}$.

As an example, consider fig. 7. There are $N_{i}$ D-branes wrapping a chain of four minimal spheres $M_{i}, i=1, \ldots 4$ connecting two $(1,0)$ branes and two $(0,1)$ branes. For every pair of spheres intersecting over an $\mathbf{S}^{1}$ we get a bifundamental scalar field, so we have matter in representations $\left(N_{i}, \bar{N}_{i+1}\right)$, where $i=5$ corresponds to the first sphere again. Note that in the $\left(N_{1}, \bar{N}_{2}\right)$ and $\left(N_{3}, \bar{N}_{4}\right)$ sector, the bifundamental scalar is not localized, and correspondingly in fig. 7 there is a family of annuli. In fact, a careful reader has probably noticed that this could have happened in the two-sphere case as well, had we not chosen judiciously the ordering of $(1,0)$ and $(0,1)$ branes in the $z$ direction of the base. In other words, we could have picked $\mu_{1}, \mu_{2}>0$ in (3.2), and we would have found a family of annuli. See fig. 7. This objection is in fact its own cure. Namely, by changing the complex structure of $X$ we could go from one configuration to the other. In fact, using the other direction in the $z$-plane we can do this in a smooth way, as the $\mu$ 's are complex, without passing through a singularity of the three manifold. On the other hand, the topological A-model amplitudes cannot depend on the complex structure moduli. As a consequence, the value of operators $\mathcal{O}\left(U_{i}, U_{i+1} ; r_{i}\right)$ cannot change in passing between the two configurations, and they are given by the annulus computation we already outlined.

This idea is rather powerful and it leads to the fact that, in all the toric cases, the only holomorphic curves are annuli connecting pairs of $\mathbf{S}^{3}$ 's along lines on the toric base (i.e. along loci of $(p, q)$ 5-branes). The argument for this is extremely simple: as we explained before, we can deform the theory to a generic point in the complex structure 
moduli space, where it is manifest that the only holomorphic curves are annuli. By the fact that topological A-model amplitudes do not depend on complex structure moduli, we can immediately conclude that only annuli contribute to topological string amplitudes. Let us now explain why this preserves only annuli. At a generic point of the complex structure moduli space (which we can always choose), the $T^{2}$ fibers degenerate over a set of points in the $z$ plane, and the three-cycles $M_{i}$ in $X$ project to lines connecting them, which are generically not aligned. Recall that the holomorphic curves project to points in the $z$ plane. This means that the "large" holomorphic curves must project to points in the $z$-plane (as discussed in section 2) where different $M_{i}$ 's intersect, and for a generic choice of complex structure these are the points where the $T^{2}$ fibers degenerate. In other words, we are left only with annuli over loci where the $T^{2}$ fibration degenerates, as we wished to show日.

\section{D-branes on Chains of Three-Manifolds and Knot Invariants}

In order to evaluate the A-model partition functions in these backgrounds we need a few additional pieces of data. Namely, we need to know how the different knots are linked, in particular their linking numbers $\operatorname{lk}\left(\gamma_{i}, \gamma_{j}\right)$, and also what is their framing the self linking number of each of $\gamma_{i}$ 's. As it is explained in 36 40, the framing is a rather subtle effect from the point of view of Chern-Simons theory, having to do with the fact that in evaluating expectation values of Wilson loop operator associated to the knot, one encounters certain ambiguities in the calculation. These are akin to a choice of point-splitting regularisation, since to calculate the self-linking number in a way that is consistent with topological invariance one must choose a "framing" by thickening a knot into a ribbon. Different framings differ by adding twists to the ribbon, the framing itself being defined as the linking number of the two edges of the ribbon. The Wilson loop operators are not invariant under the change of framing. We will show below that different choices of framing correspond in the present context to different target space geometries. The role of framing in topological string theory was discovered in [7] in a closely related context and studied subsequently in [8,41,42].

6 Note that at a generic point in complex structure moduli space, $M_{i}$ are not mutually supersymmetric, and when we add D-branes supersymmetry is broken. However, for topological A-model amplitudes to make sense we do not require supersymmetry, and D-branes need only be Lagrangian, which holds for any complex structure. 


\subsection{Rewriting $\mathcal{O}$}

Before we proceed, it is key to note that there is an illuminating way to write the operator $\mathcal{O}\left(U_{1}, U_{2} ; r\right)$, by using the techniques of [3]. If we expand the exponential explicitly, we get:

$$
1+\sum_{h=1}^{\infty} \sum_{n_{1}, \cdots, n_{h}} \frac{1}{h !} \frac{\mathrm{e}^{-r \sum_{i=1}^{h} n_{i}}}{n_{1} \cdots n_{h}} \operatorname{Tr} U_{1}^{n_{1}} \cdots \operatorname{Tr} U_{1}^{n_{h}} \operatorname{Tr} U_{2}^{-n_{1}} \cdots \operatorname{Tr} U_{2}^{-n_{h}}
$$

Now we write the $h$-uples $\left(n_{1}, \cdots, n_{h}\right)$ in terms of a vector $\vec{k}$, as in [3]: $k_{i}$ is the number of $i$ 's in $\left(n_{1}, \cdots, n_{h}\right)$. Taking into account that there are $h ! / \prod_{j} k_{j} ! h$-uples that give the same vector $\vec{k}$, and that $n_{1} \cdots n_{h}=\prod_{j} j^{k_{j}}$, we find that (4.1) equals

$$
1+\sum_{\vec{k}} \frac{\mathrm{e}^{-\ell r}}{z_{\vec{k}}} \Upsilon_{\vec{k}}\left(U_{1}\right) \Upsilon_{\vec{k}}\left(U_{2}^{-1}\right)
$$

where $z_{\vec{k}}=\prod_{j} k_{j} ! j^{k_{j}}$,

$$
\Upsilon_{\vec{k}}(U)=\prod_{j=1}^{\infty}\left(\operatorname{Tr} U^{j}\right)^{k_{j}},
$$

and $\ell=\sum_{j} j k_{j}$. Now, Frobenius formula tells us that

$$
\operatorname{Tr}_{R}(U)=\sum_{\vec{k}} \frac{1}{z_{\vec{k}}} \chi_{R}(C(\vec{k})) \Upsilon_{\vec{k}}(U)
$$

Using this together with orthonormality of the characters gives immediately that

$$
\mathcal{O}\left(U_{1}, U_{2} ; r\right)=\sum_{R} \operatorname{Tr}_{R} U_{1} \mathrm{e}^{-\ell r} \operatorname{Tr}_{R} U_{2}^{-1}
$$

where $\ell$ is the number of boxes in the Young tableau of $R$ and the sum is a sum over all representations, including the trivial one. We remind the reader that $U_{i}$ is a Wilson line in the three-manifold $M_{i}$. Notice that this operator is the cylinder propagator for a twodimensional gauge theory [43] 44], in which $r$ plays the role of time and the Hamiltonian is given by the first Casimir of $U(N)$ (which counts precisely the number of boxes $\ell$ of a representation). 


\subsection{Framing}

One of the key ideas used in [36] is that one can cut the manifold up into pieces on which one can solve the theory, and then glue them back together. Central to the story is also the relation of the Hilbert space of Chern-Simons theory with the space of conformal blocks of WZW models. Recall that all manifolds $M_{i}$ in our geometry can be obtained by gluing together solid tori with a diffeomorphism identification of the boundary. Associated to the boundary $T^{2}$ we have a finite dimensional Hilbert space, and a basis of states is labeled by the representations of the affine Lie algebra [36]. We will denote this basis by $|R\rangle$. The dual Hilbert space has a basis $\langle\bar{R}|$, where $\bar{R}$ denotes the representation conjugate to $R$. The dual pairing is simply $\left\langle\bar{R}_{1} \mid R_{2}\right\rangle=\delta_{R_{1} R_{2}}$. Notice that $|R\rangle$ can be computed by the path integral on a solid torus with insertion of a Wilson line in representation $R$ around the cycle that is non-trivial in homology. The corresponding state in the dual Hilbert space $\langle\bar{R}|$ is obtained by doing the same path integral but over the manifold with opposite orientation.

In the context of Chern-Simons theory with no insertions, because the diffeomorphism of the boundary induces a linear transformation of the Hilbert space, one can think about the path integral on $M$ in terms of the path integrals on two solid tori, that are then glued together with an $\operatorname{SL}(2, \mathbf{Z})$ matrix $V_{M}$ that specifies $M$. Since we are making no insertions, the state associated to each of the solid tori is the vacuum $|0\rangle$ (corresponding to the trivial representation), and the partition function of Chern-Simons theory on $M$ is $Z(M)=\left\langle 0\left|V_{M}\right| 0\right\rangle$.

In the problem at hand, we are interested in the Chern-Simons amplitude not in the vacuum but in the presence of Wilson lines. The gluing procedure that gives the partition function can be generalized to this setting, since the role of the insertions will simply be that the solid tori give rise to states $|R\rangle$ with arbitrary $R$. In our problem we have insertions of operators $\mathcal{O}\left(U_{i}, U_{j} ; r\right)$ corresponding to annuli connecting the two manifolds. Each annulus is attached to the $M_{i}$ 's either on its left or the right "half", and by (4.5) we can regard it as carrying a Wilson line in an arbitrary representation $R$ of the gauge group on the right half, and the conjugate representation $\bar{R}$ in the left half. We also have to sum over all representations. For example, in the two-sphere case, there is a knot on the right half of $M_{1}$ and the left half of $M_{2}$. We thus have

$$
Z\left(M_{1}, \operatorname{Tr}_{R} U\right)=\left\langle 0\left|V_{M_{1}}\right| R\right\rangle,
$$


and

$$
Z\left(M_{2}, \operatorname{Tr}_{R} U^{-1}\right)=\left\langle\bar{R}\left|V_{M_{2}}\right| 0\right\rangle
$$

where the $\operatorname{Tr}_{R} U, \operatorname{Tr}_{R} U^{-1}$ mean that we do the path integral with the insertion of these operators. Thus, by using (4.5) and the above gluing techniques, the full amplitude (3.4) can be written as:

$$
Z=\sum_{R}\left\langle 0\left|V_{M_{1}}\right| R\right\rangle e^{-\ell r}\left\langle\bar{R}\left|V_{M_{2}}\right| 0\right\rangle
$$

Note that were it not for the weight of $e^{-\ell r}$, we could use the resolution of the identity $\sum_{R}|R\rangle\langle\bar{R}|=\mathbf{1}$ and the operator insertion in (4.6) would correspond to a surgery operation that glues together $M_{1}$ and $M_{2}$. The resulting manifold $M_{1} \# M_{2}$ would have gluing matrix

$$
V_{M_{1} \# M_{2}}=V_{M_{1}} V_{M_{2}}
$$

This corresponds to the geometric fact that, when $r=0$, the two special Lagrangian three-spheres that we called $M_{1}$ and $M_{2}$ are exactly degenerate with $M_{1} \# M_{2}=\mathbf{S}^{2} \times \mathbf{S}^{1}$ that is their sum in homology. But instead we have a finite $r$-time propagation with a Hamiltonian that counts the numbers of boxes. Namely, insertion of the operator $\mathcal{O}$ corresponds to cutting off the right half of $M_{1}$ in the vacuum and the left half of $M_{2}$, and gluing in instead

$$
\mathcal{O}\left(U_{1}, U_{2} ; r\right)=\sum_{R}|R\rangle e^{-\ell r}\langle\bar{R}|
$$

In the case of fig. $6, M_{1}$ and $M_{2}$ are $\mathbf{S}^{3}$ 's with canonical framing. Since $\alpha=(1,0)$ degenerates in the left half of $M_{1}$ and $\beta=(0,1)$ in its right half, and $\alpha$ and $\beta$ are exchanged for $M_{2}$, the gluing matrices are $V_{M_{1}}=S=V_{M_{2}}^{-1}$, where $S=\left(\begin{array}{cc}0 & -1 \\ 1 & 0\end{array}\right)$. Note that standard surgery gives $\mathbf{S}^{2} \times \mathbf{S}^{1}$ with partition function equal to one, as expected. The amplitude (4.6) receives contributions from unknots on $M_{1}$ and $M_{2}$ in representation $R$.

Note that the transformation that changes the framing of the three-manifold affects the Wilson loop amplitudes. The diffeomorphism by $T^{n}$ on the boundary of the solid torus with a Wilson loop in representation $R$ in the center, adds $n$ twists to the "ribbon" that frames the knot. The change of framing acts on the Wilson loop amplitude by

$$
T|R\rangle=\mathrm{e}^{2 \pi i\left(h_{R}-c / 24\right)}|R\rangle,
$$


where $c$ is the central charge of the current algebra, and $h_{R}$ is the conformal weight of the WZW primary field in representation $R$. Recall that $h_{R}$ is given by

$$
h_{R}=\frac{\Lambda_{R} \cdot\left(\Lambda_{R}+\rho\right)}{2(k+N)}
$$

where $\Lambda_{R}$ is the highest weight of $R$ and $\rho$ is the Weyl vector. The numerator $C_{R}=$ $\Lambda_{R} \cdot\left(\Lambda_{R}+\rho\right)$ is the quadratic Casimir of the representation. While there is no natural choice of framing, there is a canonical choice at least on $\mathbf{S}^{3}$ and this corresponds to zero self-linking number. In the above example, both unknots on $\mathbf{S}^{3}$ were framed canonically. Below, we will see that other choices of framing arise as well.

\subsection{Linking}

The considerations above completely determine the gluing matrices, up to irrelevant framings that do not affect the amplitude by other than renormalization of $r$. Therefore, the linking of the different knots should be determined as well. Let us discuss this issue with a concrete example.

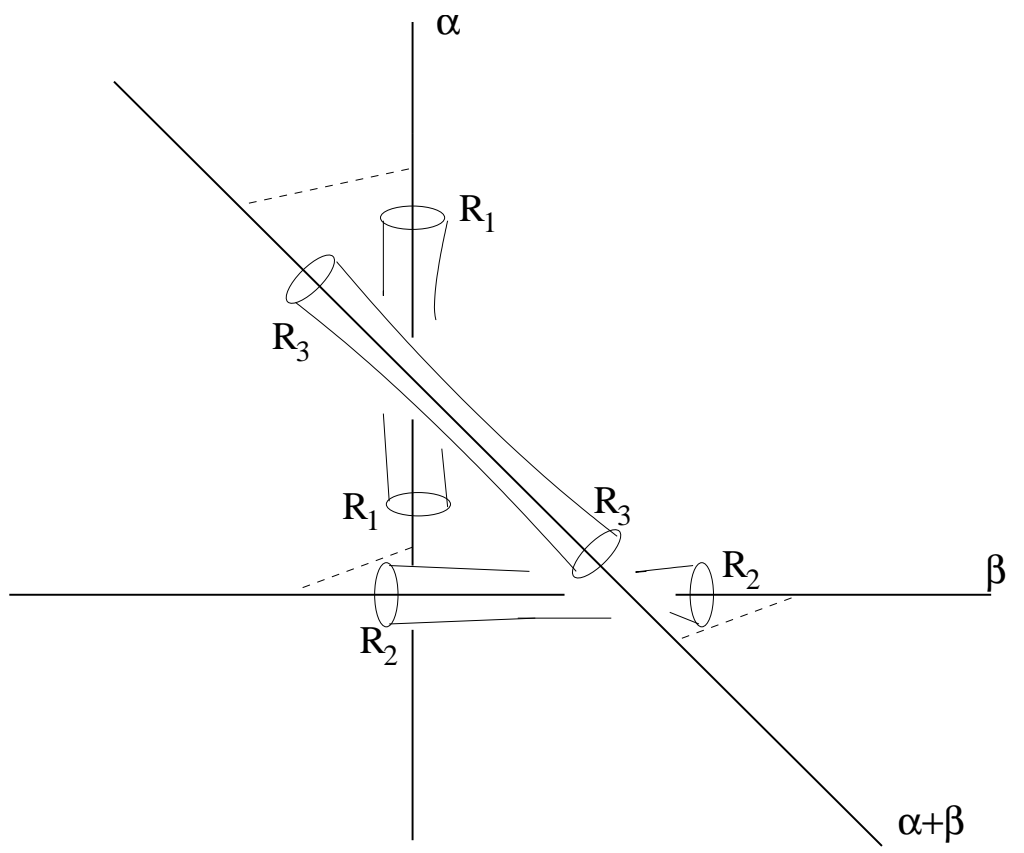

Fig. 8 In the figure there is a chain of three minimal spheres connecting $(1,0)$, $(0,1)$ and $(1,-1)$ branes, with branes wrapped on each sphere. 
The three-spheres in fig. 8 form a necklace where each is intersecting the other two over an $\mathbf{S}^{1}$, so we get scalar fields in the bifundamental, and integrating them out leaves us with the annuli shown in the figure. The path integral of the A-model in this background involves four Chern-Simons theories on a chain of four three-spheres connected with annuli:

$$
Z=\int \prod_{i=1}^{3} \mathcal{D} A_{i} e^{S_{\mathrm{CS}}\left(A_{i}\right)} \mathcal{O}\left(U_{1}, U_{2} ; r_{1}\right) \mathcal{O}\left(U_{2}, U_{3} ; r_{2}\right) \mathcal{O}\left(U_{3}, U_{1} ; r_{3}\right)
$$

There are two unknots on each three-sphere, and the amplitude will depend on their linking, in addition to their framing. Just as above, we can use (4.5) to write this in a more transparent form

$$
Z=\sum_{R_{1}, R_{2}, R_{3}}\left\langle\bar{R}_{1} \mid V_{M_{3}} R_{3}\right\rangle e^{-\ell_{3} r_{3}}\left\langle\bar{R}_{3} \mid V_{M_{2}} R_{2}\right\rangle e^{-\ell_{2} r_{2}}\left\langle\bar{R}_{2} \mid V_{M_{1}} R_{1}\right\rangle e^{-\ell_{1} r_{1}} .
$$

Since the D-branes go around the loop from one degeneration locus to the other and then back to the first one, we must have

$$
V_{M_{3}} V_{M_{2}} V_{M_{1}}=1
$$

This will hold generally whenever there are closed loops with D-branes in the toric diagram.

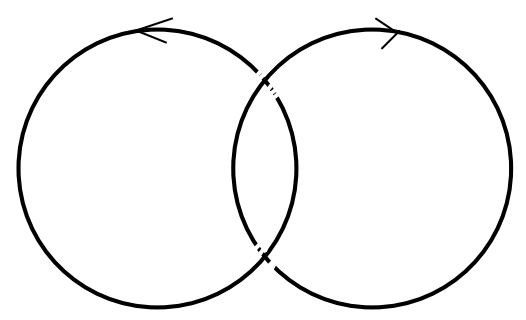

Fig. 9 The Hopf link with linking number $\mathrm{lk}=+1$.

Looking at fig. 8 we can read off,

$$
V_{M_{1}}=S^{-1}, \quad V_{M_{2}}=S T^{-1} S, \quad V_{M_{3}}=T S^{-1},
$$

so that the last factor of (4.8) is given by

$$
Z\left(M_{1}, \mathcal{L}\left(R_{2}, R_{1}\right)\right)=\left\langle\bar{R}_{2}\left|S^{-1}\right| R_{1}\right\rangle=S_{R_{1} R_{2}}^{-1}
$$


This means that $M_{1}$ is a three-sphere with two unknots that are linked into a Hopf link $\mathcal{L}\left(R_{2}, R_{1}\right)$ of linking number $\mathrm{lk}=1$ (see fig. 9). Namely, as we have seen previously, $\mathbf{S}^{3}$ is obtained by identifying two solid tori up to an $S$ transformation that exchanges the $\alpha$ and the $\beta$ cycles of the $T^{2}$. If $\beta$ is the nontrivial cycle of the solid $T^{2}$, and along this cycle one has a knot in representation $R_{1}$ and another one in representation $R_{2}$, then the $S^{-1}$ transformation results in two unknots with zero framing (this does not add any twists to the ribbons that frame the knots), but which are linked in a Hopf link with linking number $\mathrm{lk}=1$ (an $S$ transformation would give a Hopf link with linking number -1). Similarly, using $S T^{-1} S=T S^{-1} T$, we see that $M_{2}$ has a Hopf link with two knots of framing +1 .

$$
Z\left(M_{2}, \mathcal{L}\left(R_{3}, R_{2}\right)\right)=\left\langle\bar{R}_{3}\left|T S^{-1} T\right| R_{2}\right\rangle
$$

Finally, $M_{3}$ is a three-sphere with a Hopf link whose components are an unknot carrying representation $R_{1}$ and with framing +1 , and an unknot carrying representation $R_{3}$ with canonical framing:

$$
Z\left(M_{3}, \mathcal{L}\left(R_{1}, R_{3}\right)\right)=\left\langle\bar{R}_{1}\left|T S^{-1}\right| R_{3}\right\rangle .
$$

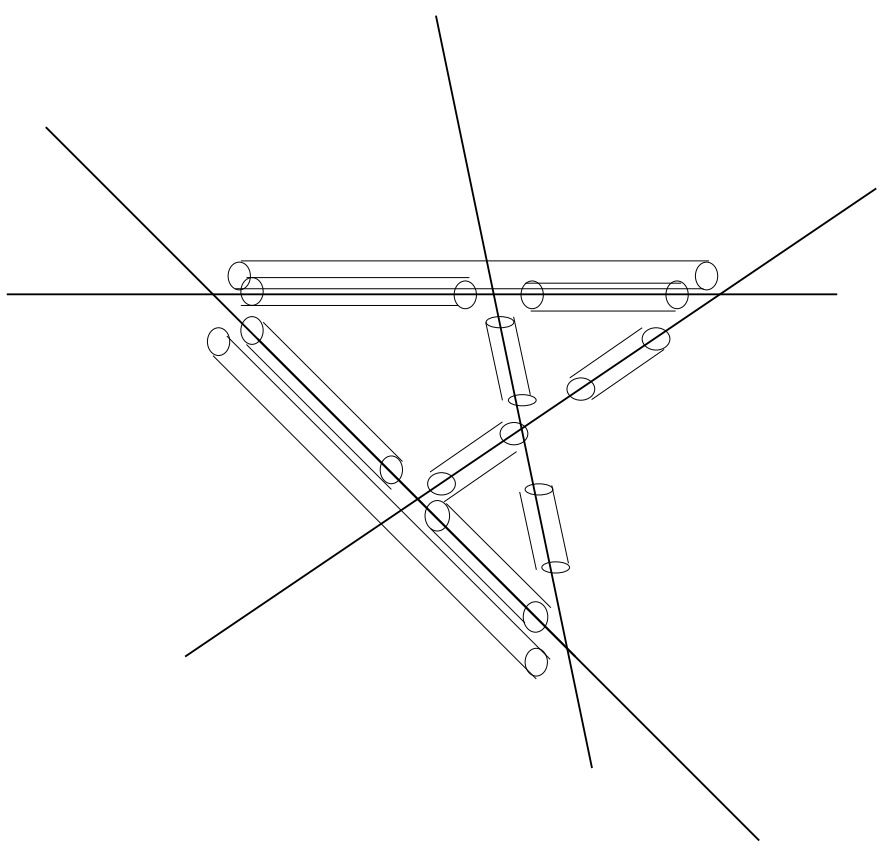

Fig. 10 The amplitude associated to this geometry can be interpreted in terms of a lattice model. The annuli correspond to states of the lattice. The 3-manifolds correspond to the interaction vertices. The figure shows the annuli on the "primitive" edges and some "non-primitive" ones. 


\subsection{Lattice model interpretation}

The models discussed above clearly generalize to more complicated geometries like the one depicted in fig. 10, where we have suppressed one direction of the base. The rules for computing the amplitudes should be clear from the previous discussion:

1) The model has states associated to all the edges of the lattice. Some of the edges are "primitive" (connecting nearest neighbor nodes) and some are "non-primitive" (connecting other nodes but always along the straight lines of the lattice) The states are labeled by representations of the affine Lie algebra (i.e. a state in the Hilbert space of $T^{2}, \mathcal{H}$ ). To each state on the $i$-th edge we associate a weight $\mathrm{e}^{-\ell r_{i}}$, where $r_{i}$ is the length of the corresponding edge and the $\ell$ is the number of boxes in the corresponding representation.

2) To every vertex we associate a linear operator. This linear operator is obtained by computing matrix elements like the ones depicted below:

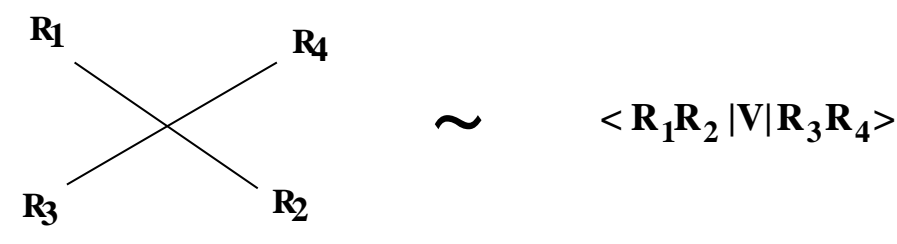

Fig. 11 The four-point vertex.

Here, $R_{1}, R_{2}$ and $R_{3}, R_{4}$ are the two pairs of representations corresponding to the collinear edges. A state $\left|R, R^{\prime}\right\rangle$ is obtained by doing the Chern-Simons path integral over the solid torus with two parallel Wilson lines inserted along its nontrivial cycle, in representations $R, R^{\prime} . V$ is the gluing matrix, as explained before. As is well known, using the fusion rules of the WZW theory, we can write

$$
\left|R, R^{\prime}\right\rangle=\sum_{R^{\prime \prime}} N_{R R^{\prime}}^{R^{\prime \prime}}\left|R^{\prime \prime}\right\rangle
$$

where the fusion coefficients are given by the Verlinde formula 445]

$$
N_{R R^{\prime}}^{R^{\prime \prime}}=\sum_{Q} \frac{S_{R Q} S_{R^{\prime} Q} S_{R^{\prime \prime} Q}^{-1}}{S_{0 Q}},
$$

Using this we can write the four-point vertex as

$$
\left\langle\bar{R}_{1}, \bar{R}_{2}|V| R_{3}, R_{4}\right\rangle=\sum_{Q, Q^{\prime}} N_{R_{1} R_{2}}^{Q} V_{Q Q^{\prime}} N_{R_{3} R_{4}}^{Q^{\prime}},
$$


where $V$ denotes the corresponding modular transformation matrix. Notice that, although there are four primitive edges ending on each vertex, one can have many non-primitive edges ending on the same vertex. In that case, we will have matrix elements of the form

$$
\left\langle\bar{R}_{1}, \cdots, \bar{R}_{n}|V| R_{1}^{\prime}, \cdots, R_{m}^{\prime}\right\rangle
$$

where the in- and out-states can be evaluated by a repeated use of the fusion rules. In (4.15), $\bar{R}_{1}, \cdots, \bar{R}_{n}$ correspond to a set of collinear edges, and $R_{1}^{\prime}, \cdots, R_{m}^{\prime}$ to the other set. As explained before the solid tori are glued together by an $\operatorname{SL}(2, \mathbf{Z})$ matrix $V$ that is computed as in (2.3).

3) Since there are edges that go off to infinity, there are boundary conditions: the state on these edges is always the trivial representationt.

4) The amplitude is the product of the linear operators over all the vertices, together with the weights associated to the connecting edges. At the end we sum over all representations on each link.

\section{Large $N$ Duality}

As was recently demonstrated [24], one can derive the large $N$ duality conjecture of Chern-Simons on $\mathbf{S}^{3}$ with topological strings [1]. In this derivation one starts with the linear sigma model description of the closed string side and finds that in some limit the theory develops Coulomb and Higgs branch. The Coulomb branch plays the role of holes in the dual Chern-Simons description.

The models we are considering here all admit a linear sigma model description [46], as discussed in [47]. Thus one can start from the gravity side, and go to the point on moduli for each $U(1)$ gauge factor and repeat the analysis of [24], which should lead to topological open string description with $N_{i}$ D-branes wrapped around $\mathbf{S}_{i}^{3}$. The analysis we did for the open string demonstrated that this open string can in turn be written in terms of some link observables in the product of $U\left(N_{i}\right)$ Chern-Simons theories. Thus we find the general prediction that

$$
F_{\text {closed }}\left(t_{i}, r_{a}^{\prime}\right)=F_{\text {open }}\left(N_{i} g_{s}, r_{a}\right)
$$

7 It would also be interesting to put periodic boundary conditions and interpret them as partially compact Calabi-Yau models. 
where by $F_{\text {open }}$ we mean the open string amplitude with link observables inserted, and the $r_{a}$ correspond to sizes of the annuli. In this equation, the $t_{i}$ on the closed string side are the Kähler moduli of the blowups corresponding to where the $\mathbf{S}_{i}^{3}$ were, and $t_{i}=N_{i} g_{s}$. As

we will see later, $r_{a}^{\prime}=r_{a}-\frac{1}{2}\left(t_{a 1}+t_{a 2}\right)$ where $t_{a i}$ denote the Kähler moduli associated to the two ends of the annulus $a$. It would be interesting to repeat in detail the analysis of [24] for the case at hand and thus obtain these shifts directly.

\section{Closed String Localization}

In this section we argue that the large $N$ duality proposal given in the previous section is in accord with localization ideas in computation of the closed string invariants.

It was suggested in 48 that one can use circle actions to localize closed topological string amplitudes. The final answer takes the form of sum over certain graphs with nodes corresponding to genus $g$ Riemann surfaces. This idea has been further developed 49. and applied to some concrete examples in [30] (at genus zero) and [29] (for higher genus). The geometry of the localization is very much related to the $(p, q)$-brane graphs we have in our setup. Basically one ends up with sums over graphs whose links correspond to intervals in the $(p, q)$ 5-brane web connecting adjacent vertices. These correspond to rational curves in the closed string side, and in the gauge theory setup they correspond to annuli. Moreover one is instructed to consider all genera computation on each node, which corresponds to mapping the whole Riemann surface to that point on the toric geometry. This ends up with a particular computation of a characteristic class on the moduli space of Riemann surfaces, that in particular depends on which links have been used in the graph. This seems to match naturally with the Chern-Simons computation, where each node is replaced by open string Riemann surfaces captured by Chern-Simons, coupled to each other through the Wilson loop expectation values coming from annuli. It is as if the Chern-Simons theory is computing directly the relevant characteristic classes on moduli of Riemann surfaces. This is not at all surprising, in light of the observations in [8] where one can use the framing dependence of unknot in Chern-Simons theory to compute all intersection numbers of Mumford classes with up to three Hodge classes, which is what the closed string side computes 41. It would be extremely interesting to make this connection with Kontsevich integral more precise and reduce the statement of the equivalence to some concrete computation at each node, which is being done using Chern-Simons gauge theory. Incidentally this is in the same spirit of the current methods of computation of these 
invariants where one uses Kontsevich's results on matrix realization of Mumford classes [50] together with certain results of Faber [51]. However, the Chern-Simon gauge theory is a more natural realization of this computation.

\section{Closed String Invariants from Chern-Simons Theory}

In this section we will show that the large $N$ duality proposed in section 5 is a powerful way to compute closed string topological A-model amplitudes for local Calabi-Yau manifolds, in terms of Chern-Simons amplitudes. In particular we will consider examples of $\mathbb{P}^{2}$ blown up at three points $\left(\mathbb{B}_{3}\right.$ del Pezzo), and $\mathbb{P}^{1} \times \mathbb{P}^{1}$ blown up at four points. Since the size of the blow ups are proportional to the rank of the corresponding dual gauge group, we can also consider the limit where the blown up $\mathbb{P}^{1}$ s have infinite size by considering the $N_{i} \rightarrow \infty$ limit. This in particular leads to computation of topological strings for $\mathbb{P}^{2}$ and $\mathbb{P}^{1} \times \mathbb{P}^{1}$ inside a Calabi-Yau threefold.

\subsection{Chern-Simons Invariants of Unknots and Hopf Links}

The toric geometries that we have described involve framed unknots and Hopf links, therefore in the evaluation of the Chern-Simons amplitudes we will need the invariants of the unknot and the Hopf link in arbitrary representations of $S U(N)$. In this section we give precise formulae for these invariants. Our notation is as follows: $W_{R_{1}, R_{2}}(\mathcal{L})$ denotes the vacuum expectation value in Chern-Simons theory corresponding to the link $\mathcal{L}$ with components $\mathcal{K}, \mathcal{K}^{\prime}$ :

$$
W_{R_{1}, R_{2}}(\mathcal{L})=\left\langle\operatorname{Tr}_{R_{1}}\left(U_{1}\right) \operatorname{Tr}_{R_{2}}\left(U_{2}\right)\right\rangle
$$

where $U_{1}, U_{2}$ are the holonomies of the gauge field around the knots $\mathcal{K}$ and $\mathcal{K}^{\prime}$, respectively. If, say $R_{2}=\cdot$ is the trivial representation, the vev (7.1) becomes the vev of the knot $\mathcal{K}$ (the second knot disappears), and we will denote this vev by $W_{R}(\mathcal{K})$. The vacuum expectation values denoted by $\langle\cdot\rangle$ are normalized, so that they denote the path integral with insertions and divided by the partition function (in other words, the vev of the identity operator is one). Of course the duality also cares for the overall normalization (i.e., the vacuum energy) and this we will put in at the end of the computation. We also recall our notation for the Chern-Simons variables:

$$
q=\exp \left(\frac{2 \pi i}{k+N}\right), \quad \lambda=q^{N} .
$$


It is well-known that the Chern-Simons invariant of the unknot in an arbitrary representation $R$ is given by the quantum dimension of $R$ :

$$
W_{R}=\frac{S_{0 R}}{S_{00}}=\operatorname{dim}_{q} R
$$

The explicit expression for $\operatorname{dim}_{q} R$ is as follows. Let $R$ be a representation corresponding to a Young tableau with row lengths $\left\{\mu_{i}\right\}_{i=1, \cdots, d(\mu)}$, with $\mu_{1} \geq \mu_{2} \geq \cdots$, and where $d(\mu)$ denotes the number of rows. Define the following $q$-numbers:

$$
\begin{aligned}
{[x] } & =q^{\frac{x}{2}}-q^{-\frac{x}{2}}, \\
{[x]_{\lambda} } & =\lambda^{\frac{1}{2}} q^{\frac{x}{2}}-\lambda^{-\frac{1}{2}} q^{-\frac{x}{2}} .
\end{aligned}
$$

Then, the quantum dimension of $R$ is given by

$$
\operatorname{dim}_{q} R=\prod_{1 \leq i<j \leq d(\mu)} \frac{\left[\mu_{i}-\mu_{j}+j-i\right]}{[j-i]} \prod_{i=1}^{d(\mu)} \frac{\prod_{v=-i+1}^{\mu_{i}-i}[v]_{\lambda}}{\prod_{v=1}^{\mu_{i}}[v-i+d(\mu)]}
$$

The quantum dimension is a Laurent polynomial in $\lambda^{ \pm \frac{1}{2}}$ whose coefficients are rational functions of $q^{ \pm \frac{1}{2}}$. In what follows in some cases we will also be interested in the leading power of $\lambda$ in the above expression. It is easy to see that this power is $\ell / 2$, where $\ell=\sum_{i} \mu_{i}$ is the total number of boxes in the representation $R$, and the coefficient of this power is the rational function of $q^{ \pm \frac{1}{2}}$

$$
q^{\kappa_{R} / 4} \prod_{1 \leq i<j \leq d(\mu)} \frac{\left[\mu_{i}-\mu_{j}+j-i\right]}{[j-i]} \prod_{i=1}^{d(\mu)} \prod_{v=1}^{\mu_{i}} \frac{1}{[v-i+d(\mu)]}
$$

where

$$
\kappa_{R}=\ell+\sum_{i=1}^{d(\mu)} \mu_{i}\left(\mu_{i}-2 i\right)
$$

This quantity is related to the quadratic Casimir of the representation. In fact, one has $\Lambda_{R} \cdot\left(\Lambda_{R}+\rho\right)=\kappa_{R}+N \ell-\ell^{2} / N$.

Let us now consider the Hopf link with linking number 1. Its invariant for representations $R_{1}, R_{2}$ is given by

$$
W_{R_{1}, R_{2}}=q^{\ell_{1} \ell_{2} / N} \frac{S_{R_{1} R_{2}}^{-1}}{S_{00}}
$$

where $\ell_{i}$ is the total number of boxes in the Young tableau of $R_{i}, i=1,2$. The prefactor $q^{\ell_{1} \ell_{2} / N}$ in (7.8) is a correction which was pointed out in [8], and is due to the fact that 
the vev $W_{R_{1}, R_{2}}$ has to be computed in the theory with gauge group $U(N)$. Although the expression for the $S$-matrix is explicitly known, it is not straightforward to write it in terms of $q$ and $\lambda$, which is what we need. We can use the Verlinde formula (4.13) giving the fusion coefficients in terms of the $S$-matrix elements, as well as the well-known identity $(S T)^{3}=S^{2}=C$, to obtain the expression

$$
W_{R_{1}, R_{2}}=\sum_{R} N_{R_{1}, R_{2}}^{R} q^{\frac{1}{2}\left(\kappa_{R}-\kappa_{R_{1}}-\kappa_{R_{2}}\right)} \operatorname{dim}_{q} R
$$

This can be also derived by using the formalism of knot operators [52] [53], and it was used in [5] to obtain the integral invariants associated to the Hopf link (we must observe, however, that in [5] the Hopf link with linking number -1 was considered). Notice that the fusion coefficients $N_{R_{1}, R_{2}}^{R}$ become, in the large $k$ limit, the Littlewood-Richardson coefficients for the tensor product $R_{1} \otimes R_{2}=\sum_{R} N_{R_{1} R_{2}}^{R} R$, and since we are evaluating the invariants at large $k, N$, to compute (7.9) we have to use these tensor product coefficients.

Another expression for the Chern-Simons invariant of the Hopf link in arbitrary representations has been recently obtained by Morton and Lukac by using skein theory [54] [55]. Let us briefly describe their result, which turns out to be very useful in order to compute the invariants. Let $\mu$ be a Young tableau, and let $\mu^{\vee}$ denote its transposed tableau (remember that this tableau is obtained from $\mu$ by exchanging rows and columns). The Schur polynomial in the variables $\left(x_{1}, \cdots, x_{N}\right)$ corresponding to $\mu$ (which is the character of the diagonal $S U(N)$ matrix $\left(x_{1}, \cdots, x_{N}\right)$ in the representation corresponding to $\mu$ ), will be denoted by $s_{\mu}$. They can be written in terms of elementary symmetric polynomials $e_{i}\left(x_{1}, \cdots, x_{N}\right), i \geq 1$, as follows [56]:

$$
s_{\mu}=\operatorname{det} M_{\mu}
$$

where

$$
M_{\mu}^{i j}=\left(e_{\mu_{i}^{\vee}+j-i}\right)
$$

$M_{\mu}$ is an $r \times r$ matrix, with $r=d\left(\mu^{\vee}\right)$. To evaluate $s_{\mu}$ we put $e_{0}=1, e_{k}=0$ for $k<0$. The expression (7.10), known sometimes as the Jacobi-Trudy identity, can be formally extended to give the Schur polynomial $s_{\mu}(E(t))$ associated to any formal power series $E(t)=1+\sum_{n=1}^{\infty} a_{i} t^{i}$. To obtain this, we simply use the Jacobi-Trudy formula (7.10), but 
where $e_{i}$ denote now the coefficients of the series $E(t)$, i.e. $e_{i}=a_{i}$. Morton and Lukac define the series $E_{\emptyset}(t)$ as follows:

$$
E_{\emptyset}(t)=1+\sum_{n=1}^{\infty} c_{n} t^{n}
$$

where the coefficients $c_{n}$ are defined by

$$
c_{n}=\prod_{i=1}^{n} \frac{1-\lambda^{-\frac{1}{2}} q^{i-1}}{q^{i}-1}
$$

They also define a formal power series associated to a tableau $\mu, E_{\mu}(t)$, as follows:

$$
E_{\mu}(t)=E_{\emptyset}(t) \prod_{j=1}^{d(\mu)} \frac{1+q^{\mu_{j}-j} t}{1+q^{-j} t}
$$

One can then consider the Schur function of the power series $(7.13), s_{\mu}\left(E_{\mu^{\prime}}(t)\right)$, for any pair of tableaux $\mu, \mu^{\prime}$, by expanding $E_{\mu^{\prime}}(t)$ and substituting its coefficients in the JacobiTrudy formula (7.10). It turns out that this Schur function is essentially the invariant we were looking for. More precisely, one has

$$
W_{R_{1}, R_{2}}=\left(\operatorname{dim}_{q} R_{1}\right)(\lambda q)^{\frac{\ell_{2}}{2}} s_{\mu_{2}}\left(E_{\mu_{1}}(t)\right)
$$

where $\mu_{1,2}$ are the tableaux corresponding to $R_{1,2}$, and $\ell_{2}$ is the number of boxes of $\mu_{2}$. More details and examples can be found in [54]. It is easy to see from (7.14) that the leading power in $\lambda$ of $W_{R_{1}, R_{2}}$ is $\left(\ell_{1}+\ell_{2}\right) / 2$, and its coefficient is given by the leading coefficient of the quantum dimension, (7.6), times a rational function of $q^{ \pm \frac{1}{2}}$ that can be easily computed by taking $\lambda \rightarrow \infty$ in $E_{\emptyset}(t)$.

As a simple example of the Morton-Lukac formula, we can compute $W_{(\square, \square)}$. In this case, $s_{\{1\}}=e_{1}$, and it is enough to expand $E_{\{1\}}(t)$ at first order,

$$
E_{\{1\}}(t)=1+\left\{1-q^{-1}+\frac{1-\lambda^{-1}}{q-1}\right\} t+\cdots,
$$

so that we obtain

$$
W_{(\square, \square)}=\left(\frac{\lambda^{\frac{1}{2}}-\lambda^{-\frac{1}{2}}}{q^{\frac{1}{2}}-q^{-\frac{1}{2}}}\right)^{2}+\lambda-1 .
$$


In the same way, one can easily find:

$$
\begin{aligned}
W_{\text {(ロ , 口 })}= & \lambda^{3} \frac{1-q^{2}+q^{3}}{\left(q^{\frac{1}{2}}-q^{-\frac{1}{2}}\right)^{3}(q+1)}-\lambda \frac{q^{-1}+1+q^{3}}{\left(q^{\frac{1}{2}}-q^{-\frac{1}{2}}\right)^{3}(q+1)} \\
& +\lambda^{-1} \frac{q^{-1}+1+q^{2}}{\left(q^{\frac{1}{2}}-q^{-\frac{1}{2}}\right)^{3}(q+1)}-\lambda^{-3} \frac{1}{\left(q^{\frac{1}{2}}-q^{-\frac{1}{2}}\right)^{3}(q+1)}, \\
W_{\text {(日, 口) }}= & \lambda^{3} \frac{q^{-2}-q^{-1}+q}{\left(q^{\frac{1}{2}}-q^{-\frac{1}{2}}\right)^{3}(q+1)}-\lambda \frac{q^{-2}+q+q^{2}}{\left(q^{\frac{1}{2}}-q^{-\frac{1}{2}}\right)^{3}(q+1)} \\
& +\lambda^{-1} \frac{q^{-1}+q+q^{2}}{\left(q^{\frac{1}{2}}-q^{-\frac{1}{2}}\right)^{3}(q+1)}-\lambda^{-3} \frac{q}{\left(q^{\frac{1}{2}}-q^{-\frac{1}{2}}\right)^{3}(q+1)},
\end{aligned}
$$

and so on. In the computations that give the invariants of $\mathbb{P}^{2}$, we only need the rational function of $q^{ \pm \frac{1}{2}}$ which multiplies the highest power in $\lambda$.

The above results are for knots and links in the standard framing. The framing can be incorporated as in [8], by simply multiplying the Chern-Simons invariant of a link with components in the representations $R_{1}, \cdots, R_{L}$, by the factor

$$
(-1)^{\sum_{\alpha=1}^{L} p_{\alpha} \ell_{\alpha}} q^{\frac{1}{2} \sum_{\alpha=1}^{L} p_{\alpha} \kappa_{R_{\alpha}}}
$$

where $p_{\alpha}, \alpha=1, \cdots, L$ are integers labeling the choice of framing for each component.

\subsection{Evaluation of the Two-Sphere Example 1}

The simplest example of how to compute a closed string amplitude from Chern-Simons theory comes from the geometry depicted in fig. 6. As explained there, this involves computing the vacuum expectation value of the operator (4.5):

$$
\mathcal{O}\left(U_{1}, U_{2} ; r\right)=\sum_{R} \operatorname{Tr}_{R} U_{1} \mathrm{e}^{-\ell r} \operatorname{Tr}_{R} U_{2}^{-1}
$$

where $U_{1}$ and $U_{2}$ are the holonomies of dynamical gauge fields (in other words, we are computing the vev in a $U\left(N_{1}\right) \times U\left(N_{2}\right)$ theory, but with the same coupling constant). We are going to discuss the operator (7.19) in a more general setting, so that $U_{1}$ is the holonomy around an arbitrary knot. We are going to assume however that $U_{2}$ is the holonomy around an unframed unknot. We now have to take the vev of this expression by doing the functional integral over both the $U\left(N_{1}\right)$ field $A_{1}$ and the $U\left(N_{2}\right)$ field $A_{2}$. Since

8 The results in this section have been obtained in collaboration with P. Ramadevi 
we are assuming that $U_{2}$ is the holonomy around an unknot with zero framing, we have that [2]

$$
\left\langle\operatorname{Tr}_{R} U_{2}^{-1}\right\rangle_{A_{2}}=\operatorname{Tr}_{R} U_{0}^{-1}
$$

where $U_{0}$ is the element in the Cartan subalgebra that corresponds to $\exp \left(2 \pi i \rho /\left(k_{2}+N_{2}\right)\right)$. Therefore, the vev with respect to the field $A_{2}$ gives

$$
\left\langle\mathcal{O}\left(U_{1}, U_{2} ; r\right)\right\rangle_{A_{2}}=\sum_{R} \operatorname{Tr}_{R} U_{1} \mathrm{e}^{-\ell r} \operatorname{Tr}_{R} U_{0}^{-1}
$$

Notice that we can regard this vev as the generating functional considered in [2], where the source takes the particular value $U_{0}^{-1}$. We can now take the vev with respect to the $A_{1}$ field, and use the results of [2] to write:

$$
\left\langle\mathcal{O}\left(U_{1}, U_{2} ; r\right)\right\rangle_{A_{1}, A_{2}}=\exp \left(\sum_{d=1}^{\infty} \frac{1}{d} \sum_{R} f_{R}\left(q^{d}, \lambda_{1}^{d}\right) \mathrm{e}^{-d \ell r} \operatorname{Tr}_{R} U_{0}^{-d}\right),
$$

This expression is valid for any framed knot along which we take the holonomy $U_{1}$ of the gauge field $A_{1}$. In this equation, $\lambda_{1}=q^{N_{1}}$ is the exponential of the 't Hooft coupling for the $U\left(N_{1}\right)$ Chern-Simons theory. We can write the exponent of the right hand side as follows,

$$
\sum_{R} f_{R}\left(q^{d}, \lambda_{1}^{d}\right) \mathrm{e}^{-\ell r} \operatorname{Tr}_{R} U_{0}^{-d}=\sum_{\vec{k}} \frac{1}{z_{\vec{k}}} f_{\vec{k}}\left(q^{d}, \lambda_{1}^{d}\right) \mathrm{e}^{-\ell r} \Upsilon_{\vec{k}}\left(U_{0}^{-d}\right),
$$

where $f_{\vec{k}}$ was introduced in [5] and is simply the character transform of the $f_{R}$. The last factor can be easily computed to be

$$
\Upsilon_{\vec{k}}\left(U_{0}^{-d}\right)=\prod_{j}\left(\frac{\lambda_{2}^{\frac{d j}{2}}-\lambda_{2}^{-\frac{d j}{2}}}{q^{\frac{d j}{2}}-q^{-\frac{d j}{2}}}\right)^{k_{j}}
$$

where $\lambda_{2}=q^{N_{2}}$.

The vev (7.22) can be written in a very suggestive way by using the results of [5] on Chern-Simons vevs. In particular, $f_{\vec{k}}$ has the structure:

$$
f_{\vec{k}}\left(q, \lambda_{1}\right)=\prod_{j}\left(q^{-\frac{j}{2}}-q^{\frac{j}{2}}\right)^{k_{j}} \sum_{g, Q} n_{\vec{k}, g, Q}\left(q^{-\frac{1}{2}}-q^{\frac{1}{2}}\right)^{2 g-2} \lambda_{1}^{Q} .
$$

Therefore, one finds for (7.22):

$$
\begin{aligned}
& \log \left\langle\mathcal{O}\left(U_{1}, U_{2} ; r\right)\right\rangle_{A_{1}, A_{2}}= \\
& \sum_{d=1}^{\infty} \sum_{g, Q} \frac{1}{d}\left(q^{-\frac{d}{2}}-q^{\frac{d}{2}}\right)^{2 g-2} \sum_{\vec{k}} \frac{n_{\vec{k}, g, Q}}{z_{\vec{k}}} \mathrm{e}^{-d \ell r} \prod_{j}\left(\lambda_{2}^{-\frac{d j}{2}}-\lambda_{2}^{\frac{d j}{2}}\right)^{k_{j}} \lambda_{1}^{d Q}
\end{aligned}
$$


This has the structure of the free energy for a closed string [57]:

$$
\sum_{d=1}^{\infty} \sum_{g, m} n_{\vec{m}}^{g} \frac{1}{d}\left(2 \sin \frac{d g_{s}}{2}\right)^{2 g-2} \mathrm{e}^{-d \vec{m} \cdot \vec{t}},
$$

provided one finds the appropriate relation between the closed string Kähler parameters $\vec{t}$, and the Kähler parameters for the open string appearing in (7.26). We will find the precise relation in the examples below. We also have to show that the expansion in (7.26) involves integers in a manifest way, since in (7.26) we are dividing the integers $n_{\vec{k}, g, Q}$ by $z_{\vec{k}}$. The way to fix that is to recall that, as shown in [5], the "primitive" integer invariants are not $n_{\vec{k}, g, Q}$, but $\widehat{N}_{R, g, Q}$. They are related by a linear transformation involving the characters of the symmetric group,

$$
n_{\vec{k}, g, Q}=\sum_{R} \chi_{R}(C(\vec{k})) \widehat{N}_{R, g, Q}
$$

Using again the results of [5], one can show that

$$
\prod_{j}\left(\lambda^{-\frac{j}{2}}-\lambda^{\frac{j}{2}}\right)^{k_{j}}=\left(\lambda^{-\frac{1}{2}}-\lambda^{\frac{1}{2}}\right) \sum_{R} \chi_{R}(C(\vec{k})) S_{R}\left(\lambda^{-1}\right)
$$

where $S_{R}(\lambda)$ is the monomial defined in [5]: if $R$ is not a hook representation, it is zero, and if $R$ is a hook of $\ell$ boxes with $\ell-s$ boxes in the first row, then

$$
S_{R}(\lambda)=(-1)^{s} \lambda^{-\frac{\ell-1}{2}+s}
$$

Using this, we find

$$
\sum_{\vec{k}} \frac{n_{\vec{k}, g, Q}}{z_{\vec{k}}} \prod_{j}\left(\lambda_{2}^{-\frac{d j}{2}}-\lambda_{2}^{\frac{d j}{2}}\right)^{k_{j}}=\left(\lambda_{2}^{-\frac{d}{2}}-\lambda_{2}^{\frac{d}{2}}\right) \sum_{R} S_{R}\left(\lambda_{2}^{-d}\right) \widehat{N}_{R, g, Q}
$$

and this would lead to the identification

$$
\sum_{m} n_{\vec{m}}^{g} \mathrm{e}^{-\vec{m} \cdot \vec{t}}=\left(\lambda_{2}^{-\frac{1}{2}}-\lambda_{2}^{\frac{1}{2}}\right) \sum_{R, Q} e^{-\ell r} S_{R}\left(\lambda_{2}^{-1}\right) \lambda_{1}^{Q} \widehat{N}_{R, g, Q}
$$

From this expression, together with a suitable linear map between Kähler parameters $\vec{t}$ and $\left(r, N_{1} g_{s}, N_{2} g_{s}\right.$ ) (which will lead to only negative exponents for $\vec{t}$ and which will depend on the choice of knot), the integral structure on both sides is compatible and one can express the closed string integral invariants $n_{d}^{g}$ in terms of the open string integral invariants $\widehat{N}_{R, g, Q}$. In the examples we will encounter in this paper, both knots will be unknots and 
the relation will be rather simple. It is interesting to notice that, when $N=M$, i.e. both gauge groups coincide, and $r=0,(7.19)$ is the partition function of the three-manifold obtained after performing surgery on the knot where $U$ is supported (for finite $k$ and $N$ ) [36] 58] [59.

The above result can be easily generalized to more complicated situations. For example, one can consider $L$ arbitrary knots, $\mathcal{K}_{\alpha}$, where $\alpha=1, \cdots, L$, and suppose that in each of the components we have a $U\left(N_{\alpha}\right)$ Chern-Simons theory. Let us also consider $L$ unknots at zero framing $\mathcal{K}_{\alpha}$ with a $U\left(M_{\alpha}\right)$ Chern-Simons theory in each of them. If we denote by $U_{\alpha}, V_{\alpha}$ the holonomies of the $U\left(N_{\alpha}\right), U\left(M_{\alpha}\right)$ fields, respectively, one can construct the operator

$$
\mathcal{O}\left(U_{1}, \cdots, U_{L} ; V_{1}, \cdots, V_{L}\right)=\exp \left[\sum_{\alpha=1}^{L} \sum_{n=1}^{\infty} \frac{\mathrm{e}^{-n r_{\alpha}}}{n} \operatorname{Tr} U_{\alpha}^{n} \operatorname{Tr} V_{\alpha}^{-n}\right]
$$

Again it can be easily shown that $\log Z\left(U_{1}, \cdots, U_{L} ; V_{1}, \cdots, V_{L}\right)$ has the structure of the free energy for a closed topological string.

As an application of the above computation, we can evaluate (4.6) when $p=0$, corresponding to fig. 6 . In this case, $U_{1}$ is the holonomy around an unknot with trivial framing, and the only nontrivial $f_{R}$ corresponds to the fundamental representation. We then find,

$$
Z\left(M_{1}, M_{2}\right)=\exp \left(-F\left(M_{1}\right)-F\left(M_{2}\right)-F\left(M_{1}, M_{2} ; r\right)\right)
$$

where $F\left(M_{i}\right)$ is the free energy of the three-sphere $M_{i}$, and

$$
F\left(M_{1}, M_{2} ; r\right)=\log \left\langle\mathcal{O}\left(U_{1}, U_{2} ; r\right)\right\rangle_{A_{1}, A_{2}}=\sum_{d=1}^{\infty} \frac{\mathrm{e}^{-d r^{\prime}}\left(1-\mathrm{e}^{-d t_{1}}\right)\left(1-\mathrm{e}^{-d t_{2}}\right)}{d\left(2 \sin \left(d g_{s} / 2\right)\right)^{2}}
$$

Notice that, in writing (7.32), we have defined:

$$
r^{\prime}=r-\frac{t_{1}+t_{2}}{2}
$$

i.e. the parameter $r$ that appears in (7.19) has to be renormalized in order to match the closed string Kähler parameter $r^{\prime}$. We will see below other examples of this, in which the same structure (7.33) appears. This shift was first observed in [27]. 

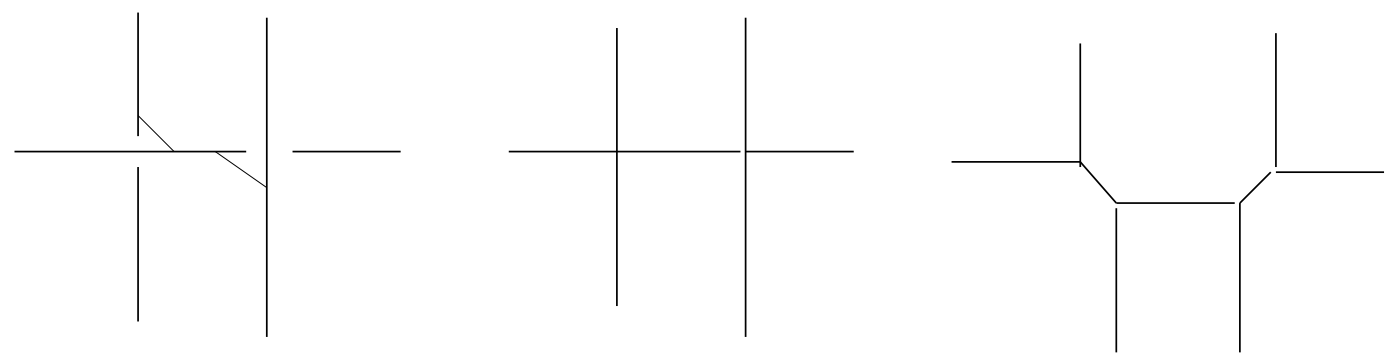

Fig. 12 After the transition, the two $\mathbf{S}^{3}$ 's that we have wrapped D-branes on disappear, and with them all of $H_{3}(X)$, so we are left with $b_{2}(X)=3$.

The dual closed string geometry is depicted in fig. 12. The two $\mathbf{S}^{3}$ 's, whose local neighborhood are $T^{*} \mathbf{S}^{3}$ 's are replaced by two $\mathbb{P}^{1}$ 's with normal bundle $\mathcal{O}(-1) \oplus \mathcal{O}(-1)$. In the total geometry, each of them intersects a $\mathbb{P}^{1}$ with $\mathcal{O} \oplus \mathcal{O}(-2)$ normal bundle.

It is not difficult to calculate the genus zero amplitude of the closed string background using mirror symmetry, as in [25], and we find

$$
F_{g=0}\left(r^{\prime}, t_{1}, t_{2}\right)=\sum_{i=1,2} \sum_{d=1}^{\infty} \frac{\mathrm{e}^{-d t_{i}}}{d^{3}}+\sum_{d=1}^{\infty} \frac{\mathrm{e}^{-d r^{\prime}}\left(1-\mathrm{e}^{-d t_{1}}\right)\left(1-\mathrm{e}^{-d t_{2}}\right)}{d^{3}},
$$

This has contribution from a single primitive curve in each of the classes $\left[r^{\prime}\right],\left[t_{i}\right],\left[r+t_{i}\right],[r+$ $\left.t_{1}+t_{2}\right]$. In fact, the only primitive curves in this geometry are rational curves, which are enumerated by the genus zero amplitude. One easy way to see this is as follows.

Note that under the duality of M-theory on $X /$ type IIB theory on $B \times S^{1}$ that we discussed in the previous sections, M2 branes wrapping holomorphic curves in $X$ that have components along the $T^{2}$ directions map to $(p, q)$ string web in type IIB string theory ending on the five-brane web (recall that M2 brane wrapping $(p, q)$ cycle of the $T^{2}$ maps to a $(p, q)$ string in type IIB string theory). The requirement for supersymmetry is that the $(p, q)$ string must be parallel to $(p, q)$ five brane, and that stings must be parallel to the plane defined by the five-branes. Compact curves in $X$ correspond to string webs ending on the five-branes, but not any string can end on any five brane - a $(p, q)$ string can only end on the $(p, q)$ five brane. These are conditions for holomorphic curves in $X$, rephrased in the IIB language (to be complete, there is also the zero force condition and string string charge conservation that must be conserved at each vertex, in addition to the junctions with five-branes).

Stated this way, it is clear from fig. 12 that there are no BPS strings of finite length in the IIB dual, and correspondingly, no curves in $X$ other than the ones counted above. 
This, together with the prediction for integrality properties of the amplitude (7.27), implies that the all genus partition function of the closed string theory is given by

$$
F=\sum_{d=1}^{\infty} \frac{e^{-d t_{1}}+e^{-d t_{2}}+e^{-d r^{\prime}}\left(1-e^{-d t_{1}}\right)\left(1-e^{-d t_{2}}\right)}{d\left(2 \sin \left(d g_{s} / 2\right)\right)^{2}}
$$

This agrees exactly with the Chern-Simons answer (7.32). Note that

$$
t_{i}=N_{i} g_{s}
$$

are two new Kähler parameters, the sizes of two-spheres that grow with $N$, replacing the $\mathbf{S}^{3}$ 's. The Kähler parameter $r^{\prime}$ was already present in the open string geometry as the size of the holomorphic annulus with boundaries on the two spheres, but we have seen that their precise relation is given by (7.33).

\section{3. $\mathcal{O}(-3) \rightarrow \mathbb{P}^{2}$}

The geometric transition in the three-sphere case is similar, and we have depicted it in fig. 3. The dual closed string geometry contains a $\mathbb{P}^{2}$ with three exceptional $\mathbb{P}^{1}$ 's touching it at three points. We can send the size of these $\mathbb{P}^{1}$ 's to infinity by taking $N_{i} \rightarrow \infty$ in our calculation to recover the $\mathbb{P}^{2}$ amplitude. The novelty in this case is that the closed string geometry has curves of arbitrarily high genus contributing to the topological A-model amplitudes, and infinitely many integer invariants are non-zero, as we will see.
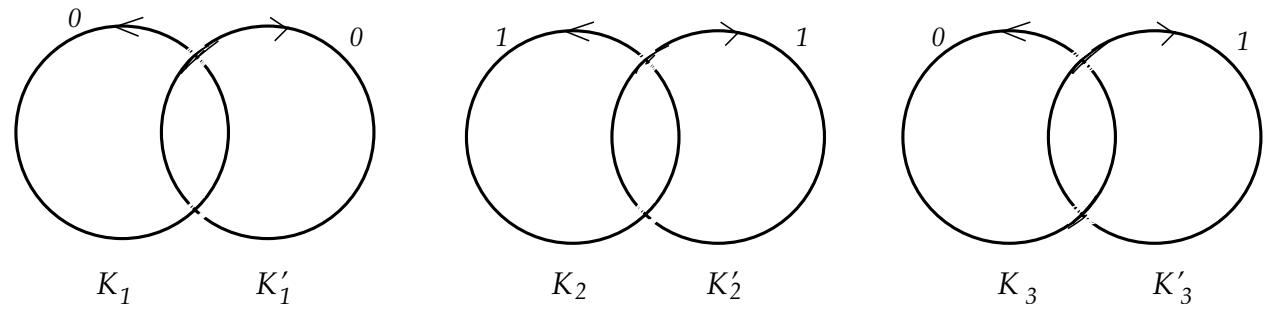

Fig. 13 The figure shows the Hopf links in the manifolds $M_{1}, M_{2}$ and $M_{3}$ respectively. The numbers indicate the framing of each knot.

We now focus on the Chern-Simons computation that gives the invariants for $\mathcal{O}(-3) \rightarrow$ $\mathbb{P}^{2}$. According to what we discussed before, the Chern-Simons scenario involves three different gauge groups, with 't Hooft parameters $t_{1}, t_{2}, t_{3}$, and with the same coupling constant $g_{s}$. Accordingly, the quantum invariants will be a rational function of $q$ and $\lambda_{i}=\mathrm{e}^{t_{i}}, i=1,2,3$. As we saw in section 5, for each Chern-Simons theory we have a 
Hopf link, and we will denote them by $\mathcal{L}_{i}$, with components $\mathcal{K}_{i}$ and $\mathcal{K}_{i}^{\prime}, i=1,2,3$. The framings can be read from (4.10), (4.12) and (4.11), and are as follows: $\mathcal{K}_{1}, \mathcal{K}_{1}^{\prime}$ and $\mathcal{K}_{3}$ have framing zero, while the remaining knots have framing $p=1$. This means that $\mathcal{L}_{1}$ is in the canonical framing, in $\mathcal{L}_{2}$ both components are framed, while in $\mathcal{L}_{3}$ only one of the components, $\mathcal{K}_{3}^{\prime}$, is framed. The free energy at all genus for the topological closed string is given by

$$
F=\log \left\{\sum_{R_{1}, R_{2}, R_{3}} \mathrm{e}^{-\sum_{i=1}^{3} \ell_{i} r_{i}} W_{R_{1}, R_{2}}\left(\mathcal{L}_{1}\right) W_{R_{2}, R_{3}}\left(\mathcal{L}_{2}\right) W_{R_{3}, R_{1}}\left(\mathcal{L}_{3}\right)\right\}
$$

The sum in (7.35) is over all possible representations, including the trivial one (and that will be denoted by ·). In this equation, $r_{i}$ are "bare" Kähler parameters that will lead to a "renormalized" Kähler parameter $r$ for the Kähler class of $\mathcal{O}(-3) \rightarrow \mathbb{P}^{2}$. The relation between $r_{i}$ and $r$ can be obtained by requiring a consistent limit $t_{i} \rightarrow \infty$ (which corresponds to the local $\mathbb{P}^{2}$ limit of the original, more complicated geometry). We will discuss this relation in a moment. Of course, there is also a piece $F\left(M_{1}\right)+F\left(M_{2}\right)+F\left(M_{3}\right)$ given by the sum of the free energies of the spheres that should be added to (7.35).

Once the "renormalized" parameter has been restored, and the limit $t_{i} \rightarrow \infty$ taken, the free energy has the structure:

$$
F=\log \left\{1+\sum_{\ell=1}^{\infty} a_{\ell}(q) \mathrm{e}^{-\ell r}\right\}=\sum_{\ell=1}^{\infty} a_{\ell}^{(c)}(q) \mathrm{e}^{-\ell r}
$$

and we will refer to $a_{\ell}^{(c)}(q)$ as to the connected coefficients. If we keep the $t_{i}$ finite, we find the free energy for a closed string propagating in $\mathbb{P}^{2}$ blown up at 3 points, which is known as $\mathbb{B}_{3}$ (a particular case of del Pezzo). The 1 inside the brackets in (7.36) corresponds to $R_{1}=R_{2}=R_{3}=$, i.e. all the representations being the trivial one. In order to extract the integral invariants from this expression, we have to recall the general structure of the closed string topological amplitudes (7.27). One can use this formula to write the integral invariants in terms of closed string amplitudes by using the Möbius function $\mu(n)$ [60]. Recall that $\mu(n)=0$ if $n$ is not square-free, and it is $(-1)^{f}$ otherwise, where $f$ is the number of factors in the prime decomposition of $n$. One finds:

$$
\sum_{g \geq 0}(-1)^{g-1} n_{d}^{g}\left(q^{-\frac{1}{2}}-q^{\frac{1}{2}}\right)^{2 g-2}=\sum_{k \mid d} \frac{\mu(k)}{k} a_{d / k}^{(c)}\left(q^{k}\right) .
$$


Therefore, if we know the coefficients $a_{\ell}^{(c)}$ for $\ell=1, \cdots, d$, we can extract the integral invariants $n_{\ell}^{g}$ for degrees $\ell=1, \cdots, d$, and for all genera. Of course, from the point of view of Chern-Simons theory, it is highly nontrivial that the coefficients $a_{\ell}^{(c)}$ extracted from (7.35) have the structure required by (7.37). In the examples discussed in the previous section, the properties of the Chern-Simons invariants derived in [2] [5] guaranteed that one obtained a closed string expansion at all degrees and genera. Here we do not have a general proof, but we will explicitly show at low degrees that again, the rather constraining structural properties of the invariants of knots and links guarantee that one finds the right structure (7.37).

Let us now look at the expansion of (7.36) at low degrees, which will also fix the relation between $r_{i}$ and $r$. First notice that, at every given degree $d$, we have a combinatorial problem of finding all possible representations $R_{1}, R_{2}, R_{3}$ such that $\ell_{1}+\ell_{2}+\ell_{3}=d$. At degree one, we have three possibilities ( $\square \cdots$ and two permutations), and we find:

$$
\mathrm{e}^{-r} a_{1}=\mathrm{e}^{-r_{1}} W_{\mathbf{\square}}\left(\mathcal{K}_{1}\right) W_{\mathbf{\square}}\left(\mathcal{K}_{3}^{\prime}\right)+\mathrm{e}^{-r_{2}} W_{\mathbf{\square}}\left(\mathcal{K}_{2}\right) W_{\mathbf{\square}}\left(\mathcal{K}_{1}^{\prime}\right)+\mathrm{e}^{-r_{3}} W_{\square}\left(\mathcal{K}_{3}\right) W_{\square}\left(\mathcal{K}_{2}^{\prime}\right) .
$$

The knots involved in this computation are just framed unknots. Since the invariant of an unknot with framing $p$ in the fundamental representation is

$$
(-1)^{p} \frac{\lambda^{-\frac{1}{2}}-\lambda^{\frac{1}{2}}}{q^{-\frac{1}{2}}-q^{\frac{1}{2}}}
$$

we see that in order to have a finite limit as $t_{i} \rightarrow \infty$, we must have:

$$
r=r_{1}-\frac{t_{1}+t_{3}}{2}=r_{2}-\frac{t_{1}+t_{2}}{2}=r_{3}-\frac{t_{2}+t_{3}}{2}
$$

so we take the limit $t_{i} \rightarrow \infty$ and at the same time $r_{i} \rightarrow \infty$ in such a way that the above combinations remain finite and equal to the closed string Kähler parameter $r$. The relation (7.40) is identical to the one we found before, in (7.33). Notice that, from the point of view of the Chern-Simons computation, this means that we have to renormalize every vev in the representations $R, R^{\prime}$ as follows,

$$
W_{R, R^{\prime}}\left(\mathcal{L}_{i}\right) \rightarrow \lambda_{i}^{-\frac{\ell+\ell^{\prime}}{2}} W_{R, R^{\prime}}\left(\mathcal{L}_{i}\right)
$$

where $\ell, \ell^{\prime}$ are the number of boxes in the representations $R, R^{\prime}$. We have assumed that the renormalized Kähler parameters corresponding to the different annuli are all equal to 
the single Kähler parameter of the local $\mathbb{P}^{2}$ geometry. As we will see later, one can take the renormalized parameters to be different to obtain a refined version of the invariants.

The conclusion of the above analysis is that, in order to recover the local $\mathbb{P}^{2}$ geometry, we have to take the limit $\lambda_{i} \rightarrow \infty$ after the renormalization factor has been introduced. We then find that, for the local $\mathbb{P}^{2}$ geometry,

$$
a_{1}(q)=-\frac{3}{\left(q^{-\frac{1}{2}}-q^{\frac{1}{2}}\right)^{2}}
$$

which gives immediately the integral invariants at degree one:

$$
n_{1}^{0}=3, \quad n_{1}^{g}=0 \text { for } g>0,
$$

indeed the right result [29] [31].

Let us do the computation at degree two. There are nine possible choices of representations that lead to this degree: $\square \cdot, \boldsymbol{\theta} \cdot$, 口ם·, and their permutations. This gives

$$
\begin{aligned}
a_{2} & =\lambda_{1}^{-1}\left(\lambda_{2} \lambda_{3}\right)^{-\frac{1}{2}} W_{(\square, \square)}\left(\mathcal{L}_{1}\right) W_{\square}\left(\mathcal{K}_{3}^{\prime}\right) W_{\square}\left(\mathcal{K}_{2}\right) \\
& +\left(\lambda_{1} \lambda_{3}\right)^{-1}\left(W_{\square}\left(\mathcal{K}_{1}\right) W_{\square}\left(\mathcal{K}_{3}^{\prime}\right)+W_{\text {日 }}\left(\mathcal{K}_{1}\right) W_{\text {日 }}\left(\mathcal{K}_{3}^{\prime}\right)\right)+\text { perms }
\end{aligned}
$$

where the permutations act cyclically as follows: $\mathcal{L}_{1} \rightarrow \mathcal{L}_{2} \rightarrow \mathcal{L}_{3}, \mathcal{K}_{1} \rightarrow \mathcal{K}_{2} \rightarrow \mathcal{K}_{3}$, $\mathcal{K}_{1}^{\prime} \rightarrow \mathcal{K}_{2}^{\prime} \rightarrow \mathcal{K}_{3}^{\prime}$. The connected coefficient can be easily computed to be,

$$
\begin{aligned}
a_{2}^{(c)} & =\lambda_{1}^{-1}\left(\lambda_{2} \lambda_{3}\right)^{-\frac{1}{2}} f_{(\square, \square)}\left(\mathcal{L}_{1}\right) W_{\square}\left(\mathcal{K}_{3}^{\prime}\right) W_{\square}\left(\mathcal{K}_{2}\right)+\text { perms } \\
& +\sum_{R=\square, \boxminus}\left(\left(\lambda_{2} \lambda_{3}\right)^{-1} f_{R}\left(\mathcal{K}_{2}^{\prime}\right) W_{R}\left(\mathcal{K}_{3}\right)+\left(\lambda_{1} \lambda_{3}\right)^{-1} f_{R}\left(\mathcal{K}_{3}^{\prime}\right) W_{R}\left(\mathcal{K}_{1}\right)\right. \\
& \left.+\left(\lambda_{1} \lambda_{2}\right)^{-1} f_{R}\left(\mathcal{K}_{2}\right) W_{R}\left(\mathcal{K}_{1}^{\prime}\right)\right) \\
& +\frac{1}{2}\left(\lambda_{1} \lambda_{3}\right)^{-1} W_{\square}^{(2)}\left(\mathcal{K}_{3}^{\prime}\right) W_{\square}^{(2)}\left(\mathcal{K}_{2}\right)+\text { perms. }
\end{aligned}
$$

We have denoted $W_{R}^{(2)}(q, \lambda)=W_{R}\left(q^{2}, \lambda^{2}\right)$. The invariant $f_{\left(R_{1}, R_{2}\right)}(\mathcal{L})$ was introduced in [5], and we recall that for $R_{1}=R_{2}=\square$, one has

$$
f_{(\square, \square)}(\mathcal{L})=W_{(\square, \square)}(\mathcal{L})-W_{\square}\left(\mathcal{K}_{1}\right) W_{\square}\left(\mathcal{K}_{2}\right),
$$

where $\mathcal{K}_{1,2}$ are the components of $\mathcal{L}$. One can also prove [5] that $f_{(\square, \square)}(\mathcal{L})$ has the structure:

$$
f_{(\square, \square)}(\mathcal{L})=\sum_{g, Q} \widehat{N}_{(\square, \square), g, Q}\left(q^{-\frac{1}{2}}-q^{\frac{1}{2}}\right)^{2 g} \lambda^{Q}
$$


so it is an even polynomial in $q^{-\frac{1}{2}}-q^{\frac{1}{2}}$. We can now show that the structure of $(7.45)$ is compatible with (7.37): the first three lines give a Laurent polynomial in $q^{-\frac{1}{2}}-q^{\frac{1}{2}}$ with even powers $\geq-2$. For the first line, this is guaranteed by (7.47), while for the second and third lines we just notice that they have the structure of (7.23) (with $d=1$ ), therefore we can use (7.25)(7.29) to write it in the desired form. In fact, the last three lines of (7.45) are precisely what we would obtain if the knots were all unlinked, and therefore the arguments of the last section guarantee that they have the right structure. Indeed, the very last line gives very precisely the two-cover of the degree one contribution, in agreement with (7.37).

In the above computation we have considered the most general geometry with four Kähler classes. In order to recover the local $\mathbb{P}^{2}$ case, we have to take $t_{i} \rightarrow \infty$. It is easy to see that in this limit the only relevant integral invariants of the links are

$$
\widehat{N}_{(\square, \square), g=0, Q=1}\left(\mathcal{L}_{1}\right)=\widehat{N}_{(\square, \square), g=0, Q=1}\left(\mathcal{L}_{2}\right)=-\widehat{N}_{(\square, \square), g=0, Q=1}\left(\mathcal{L}_{3}\right)=1 .
$$

These invariants can be computed from (7.46) and (7.16), after including the framing corrections $\mathrm{Q}$, while for the framed unknots we only need, in this limit, the invariant [8]

$$
\widehat{N}_{\text {日,g=0,Q=1}}(p=1)=1 .
$$

The relevant integral invariants with $g>0$ all vanish. We then find,

$$
a_{2}^{(c)}(q)=\frac{6}{\left(q^{-\frac{1}{2}}-q^{\frac{1}{2}}\right)^{2}}+\frac{1}{2} a_{1}\left(q^{2}\right),
$$

therefore:

$$
n_{2}^{0}=-6, \quad n_{2}^{g}=0 \text { for } g>0,
$$

again in agreement with the A and B-model computations [29] [31].

The procedure is now clear: to any given degree $d$, one has to compute the coefficient $a_{d}$ as a sum of different contributions given by the combinatorics of Young tableaux, compute the connected piece, and finally extract the multicovering contributions. Notice that, if one is just interested in the local $\mathbb{P}^{2}$ results, one can take the limit $t_{i} \rightarrow \infty$ at the beginning of the computation. In this limit we only have to keep the leading term in $\lambda$ in the Chern-Simons invariant of the Hopf link presented in section 5.1. In this way we have

9 In fact, many of the Chern-Simons invariants of the Hopf link with trivial framing can be read from section 6.2 of [5], after changing $\lambda, q \rightarrow \lambda^{-1}, q^{-1}$ due to the fact that the Hopf link considered there has the opposite linking number to the one considered here. 
a very powerful method to compute the integral invariants of the local $\mathbb{P}^{2}$ geometry that can be easily implemented in a symbolic manipulation program. The results, up to degree 12 and at all genera, are presented in the following tables:

\begin{tabular}{|c|c|c|c|c|c|c|c|c|c|}
\hline$g$ & $d=1$ & 2 & 3 & 4 & 5 & 6 & 7 & 8 & 9 \\
\hline 0 & 3 & $3-6$ & 27 & -192 & 1695 & -17064 & 188454 & -2228160 & 27748899 \\
\hline 1 & 0 & 0 & -10 & 231 & -4452 & 80948 & -1438086 & 25301295 & -443384578 \\
\hline 2 & 0 & 0 & 0 & -102 & 5430 & -194022 & 5784837 & -155322234 & 3894455457 \\
\hline 3 & 0 & 0 & 0 & 15 & -3672 & 290853 & -15363990 & 649358826 & -23769907110 \\
\hline 4 & 0 & 0 & 0 & 0 & 1386 & -290400 & 29056614 & -2003386626 & 109496290149 \\
\hline 5 & 0 & 0 & 0 & 0 & -270 & 196857 & -40492272 & 4741754985 & -396521732268 \\
\hline 6 & 0 & 0 & 0 & 0 & 21 & -90390 & 42297741 & -8802201084 & 1156156082181 \\
\hline 7 & 0 & 0 & 0 & 0 & 0 & 27538 & -33388020 & 12991744968 & -2756768768616 \\
\hline 8 & 0 & 0 & 0 & 0 & 0 & -5310 & 19956294 & -15382690248 & 5434042220973 \\
\hline 9 & 0 & 0 & 0 & 0 & 0 & 585 & -9001908 & 14696175789 & -8925467876838 \\
\hline 10 & 0 & 0 & 0 & 0 & 0 & -28 & 3035271 & -11368277886 & 12289618988434 \\
\hline 11 & 0 & 0 & 0 & 0 & 0 & 0 & -751218 & 7130565654 & -14251504205448 \\
\hline 12 & 0 & 0 & 0 & 0 & 0 & 0 & 132201 & -3624105918 & 13968129299517 \\
\hline 13 & 0 & 0 & 0 & 0 & 0 & 0 & -15636 & 1487970738 & -11600960414160 \\
\hline 14 & 0 & 0 & 0 & 0 & 0 & 0 & 1113 & -490564242 & 8178041540439 \\
\hline 15 & 0 & 0 & 0 & 0 & 0 & 0 & -36 & 128595720 & -4896802729542 \\
\hline 16 & 0 & 0 & 0 & 0 & 0 & 0 & 0 & -26398788 & 2489687953666 \\
\hline 17 & 0 & 0 & 0 & 0 & 0 & 0 & 0 & 4146627 & -1073258752968 \\
\hline 18 & 0 & 0 & 0 & 0 & 0 & 0 & 0 & -480636 & 391168899747 \\
\hline 19 & 0 & 0 & 0 & 0 & 0 & 0 & 0 & 38703 & -120003463932 \\
\hline 20 & 0 & 0 & 0 & 0 & 0 & 0 & 0 & -1932 & 30788199027 \\
\hline 21 & 0 & 0 & 0 & 0 & 0 & 0 & 0 & 45 & -6546191256 \\
\hline 22 & 0 & 0 & 0 & 0 & 0 & 0 & 0 & 0 & 1138978170 \\
\hline 23 & 0 & 0 & 0 & 0 & 0 & 0 & 0 & 0 & -159318126 \\
\hline 24 & 0 & 0 & 0 & 0 & 0 & 0 & 0 & 0 & 17465232 \\
\hline 25 & 0 & 0 & 0 & 0 & 0 & 0 & 0 & 0 & -1444132 \\
\hline 26 & 0 & 0 & 0 & 0 & 0 & 0 & 0 & 0 & 84636 \\
\hline 27 & 0 & 0 & 0 & 0 & 0 & 0 & 0 & 0 & -3132 \\
\hline 28 & 0 & 0 & 0 & 0 & 0 & 0 & 0 & 0 & 55 \\
\hline
\end{tabular}

Table 1: The integral invariants $n_{d}^{g}$ for the local $\mathbb{P}^{2}$ case. 


\begin{tabular}{|c|c|c|c|}
\hline$g$ & $d=10$ & 11 & 12 \\
\hline 0 & -360012150 & 4827935937 & -66537713520 \\
\hline 1 & 7760515332 & -135854179422 & 2380305803719 \\
\hline 2 & -93050366010 & 2145146041119 & -48109281322212 \\
\hline 3 & 786400843911 & -24130293606924 & 698473748830878 \\
\hline 4 & -5094944994204 & 210503102300868 & -7935125096754762 \\
\hline 5 & 26383404443193 & -1485630816648252 & 73613315148586317 \\
\hline 6 & -111935744536416 & 8698748079113310 & -572001241783007370 \\
\hline 7 & 395499033672279 & -42968546119317066 & 3786284014554551293 \\
\hline 8 & -1177301126712306 & 181202644392392127 & -21609631514881755756 \\
\hline 9 & 2978210177817558 & -658244675887405242 & 107311593188998164015 \\
\hline 10 & -6445913624274390 & 2074294284130247058 & -466990545532708577390 \\
\hline 11 & 12001782164043306 & -5702866358492557440 & 1791208287019324701495 \\
\hline 12 & -19310842755095748 & 13744538465609779287 & -6085017394087513680618 \\
\hline 13 & 26952467292328782 & -29157942375100015002 & 18384612378910358924791 \\
\hline 14 & -32736035592797946 & 54641056077839878893 & -49578782776769125835658 \\
\hline 15 & 34693175820656421 & -90735478019244786786 & 119723947998685791289164 \\
\hline 16 & -32151370513161966 & 133885726253316075984 & -259634731498425150837576 \\
\hline 17 & 26099440805196660 & -175976406401479949154 & 506961721474582218552270 \\
\hline 18 & -18580932613650624 & 206477591201198965488 & -893407075206205808615238 \\
\hline 19 & 11609627766170547 & -216671841840838260606 & 1424048002136300951108030 \\
\hline 20 & -6367395873587820 & 203674311322868998065 & -2057099617415644933602618 \\
\hline 21 & 3064262549419899 & -171730940091766865658 & 2697839037217627321703085 \\
\hline 22 & -1292593922494452 & 130015073789764141299 & -3217397468483821476968358 \\
\hline 23 & 477101143946277 & -88451172530198637924 & 3494176460021369389735746 \\
\hline 24 & -153692555590206 & 54098277648908454123 & -3460084190968494003073062 \\
\hline 25 & 43057471189239 & -29751302949160261398 & 3127576636374963802648718 \\
\hline 26 & -10441089412308 & 14709694749741501501 & -2582938330708242629937150 \\
\hline 27 & 2177999212647 & -6535189635435373326 & 1950461493734929553600580 \\
\hline 28 & -387688567518 & 2606677300588276035 & -1347524558332336039964082 \\
\hline 29 & 58269383541 & -932238829973577348 & 852109374825775079556606 \\
\hline 30 & -7292193288 & 298408032566091294 & -493309207337589509893062 \\
\hline 31 & 745600245 & -85297647759486510 & 261477149328500781917776 \\
\hline 32 & -60650490 & 21708810999461607 & -126876156355185161374314 \\
\hline 33 & 3773652 & -4901354114590566 & 56339101711825399890960 \\
\hline
\end{tabular}

Table 2: The integral invariants $n_{d}^{g}$ for the local $\mathbb{P}^{2}$ case (continuation). 


\begin{tabular}{|c|c|c|c|}
\hline$g$ & $d=10$ & 11 & 12 \\
\hline 34 & -168606 & 977233475777499 & -22881258328195868502320 \\
\hline 35 & 4815 & 171090302865948 & 8492649924309368930964 \\
\hline 36 & -66 & 26117674453665 & -2877665040430021956492 \\
\hline 37 & 0 & -3445690553358 & 888968505074075552261 \\
\hline 38 & 0 & 388460380746 & -249952226921825722236 \\
\hline 39 & 0 & -36878620320 & 63836429603183934921 \\
\hline 40 & 0 & 2891025822 & -14772524364719546808 \\
\hline 41 & 0 & -182125500 & 3088415413809592461 \\
\hline 42 & 0 & 8859513 & -581271967556317272 \\
\hline 43 & 0 & -312270 & 98073062075574517 \\
\hline 44 & 0 & 7095 & -14758388168491098 \\
\hline 45 & 0 & -78 & 1968679573589997 \\
\hline 46 & 0 & 0 & -231043750764510 \\
\hline 47 & 0 & 0 & 23635158339861 \\
\hline 48 & 0 & 0 & -2082988758060 \\
\hline 49 & 0 & 0 & 155790863415 \\
\hline 50 & 0 & 0 & -9693024822 \\
\hline 51 & 0 & 0 & 488072208 \\
\hline 52 & 0 & 0 & -19105426 \\
\hline 53 & 0 & 0 & 545391 \\
\hline 54 & 0 & 0 & -10098 \\
\hline 55 & 0 & 0 & 91 \\
\hline
\end{tabular}

Table 3: The integral invariants $n_{d}^{g}$ for the local $\mathbb{P}^{2}$ case (continuation).

It is interesting to compare this procedure to obtain the integer invariants with the ones based in the A and the B-model. As in the A-model computations based on localization, our procedure has to proceed degree by degree, and as the degree is increased the number of terms that contribute to $a_{d}$ grows very rapidly: to evaluate the integer invariants up to degree 12 , one has to find $a_{1}, \cdots, a_{12}$, and this involves evaluating 18239 terms in total. Degree 20 involves 943304 terms (there are 341649 terms contributing just to $\left.a_{20}\right)$. However, the number of terms seems to be substantially lower than in a localization computation (compare for example with [29]), and of course the crucial advantage of the Chern-Simons approach is that one gets the invariants for all genera. This is also its main advantage with respect to the B-model computations, which also become more and 
more difficult as the genus is increased. The B-model results for higher genera are in fact determined only up to some unknown constants, due to the holomorphic ambiguity [39], and in order to find the actual value of the invariants one has to provide the value of the integral invariants coming from A-model computations. Therefore, the computation via Chern-Simons provides another way of fixing the holomorphic ambiguity of the B-model.

Some comments on the results listed in Tables 1-3 are in order. First observe that, for a given degree $d, n_{d}^{g}$ vanishes for $g>(d-1)(d-2) / 2$. Indeed, $(d-1)(d-2) / 2$ is the genus of a nondegenerate curve of degree $d$ in $\mathbb{P}^{2}$. As shown in [31], one has in this case

$$
n_{d}^{(d-1)(d-2) / 2}=\frac{(-1)^{d(d+3) / 2}}{2}(d+1)(d+2)
$$

in full agreement with the corresponding entries in Table 1 for $d=1, \cdots, 12$. For $d>2$, we have contributions from curves with one node (therefore $g=d(d-3) / 2$ ), and the arguments of [31] give

$$
n_{d}^{d(d-3) / 2}=-(-1)^{d(d+3) / 2}\left(\begin{array}{l}
d \\
2
\end{array}\right)\left(d^{2}+d-3\right)
$$

again in full agreement with the results that we have obtained. Curves with two nodes start contributing at $d>3$, and one finds:

$$
n_{d}^{\left(d^{2}-3 d-2\right) / 2}=\frac{(-1)^{d(d+3) / 2}}{4}(d-1)\left(d^{5}-2 d^{4}-6 d^{3}+9 d^{2}+36\right)
$$

which reproduces our results for $4 \leq d \leq 12$. For curves with three nodes, the integral invariant is given by

$n_{d}^{\left(d^{2}-3 d-4\right) / 2}=-\frac{(-1)^{d(d+3) / 2}}{12}\left(-96+222 d-323 d^{2}+54 d^{3}-34 d^{4}+36 d^{5}+2 d^{6}-6 d^{7}+d^{8}\right)$,

which reproduces our results for $5 \leq d \leq 12$. For $d=4$ there are reducible curves with three nodes, and in order to reproduce $n_{4}^{0}$ one has to introduce a correction, as explained in detail in [31]. We then see that the results obtained from Chern-Simons theory are in full agreement with what is expected from the geometric interpretation of the integral invariants. Notice that we have been able to check results for very high genus, which is not easy to do in the $\mathrm{A}$ or $\mathrm{B}$ model computations. 


\section{4. $\mathbb{B}_{3}$}

In the previous subsection we have seen how to recover the integer invariants for $\mathbb{P}^{2}$ by taking the limit $t_{i} \rightarrow \infty$. Keeping the blow up parameters $t_{i}$ finite we obtain the integer invariants of the local del Pezzo $\mathbb{B}_{3}$ (we remind that $\mathbb{B}_{3}$ is the rational surface obtained from $\mathbb{P}^{2}$ by blowing up three points). We will write the generating functional for the integer invariants at genus $g$ as

$$
\mathcal{F}_{g}\left(r, t_{1}, t_{2}, t_{3}\right)=\sum_{\ell} \mathrm{e}^{-\ell r} \mathcal{F}_{\ell}^{g}\left(t_{1}, t_{2}, t_{3}\right)
$$

where

$$
\mathcal{F}_{d}^{g}\left(t_{1}, t_{2}, t_{3}\right)=\sum_{d_{1}, d_{2} d_{3}} n_{d_{1}, d_{2}, d_{3}}^{g} q_{1}^{d_{1}} q_{2}^{d_{2}} q_{3}^{d_{3}}
$$

and we have written $q_{i}=\mathrm{e}^{-t_{i}}$ (these shouldn't be confused with the Chern-Simons variable introduced before). We present the results for these generating functionals up to degree four in $\mathbb{P}^{2}$ :

$$
\begin{aligned}
\mathcal{F}_{1}^{0}= & -2\left(q_{1}+q_{2}+q_{3}\right)+q_{1} q_{2}+q_{1} q_{3}+q_{2} q_{3}, \\
\mathcal{F}_{2}^{0}= & -6+5\left(q_{1}+q_{2}+q_{3}\right)-4\left(q_{1} q_{2}+q_{1} q_{3}+q_{2} q_{3}\right)+3 q_{1} q_{2} q_{3}, \\
\mathcal{F}_{3}^{0}= & 27-32\left(q_{1}+q_{2}+q_{3}\right)+35\left(q_{1} q_{2}+q_{1} q_{3}+q_{2} q_{3}\right)+7\left(q_{1}^{2}+q_{2}^{2}+q_{3}^{2}\right) \\
& -6\left(q_{1} q_{2}^{2}+q_{1} q_{3}^{2}+q_{2} q_{1}^{2}+q_{2} q_{3}^{2}+q_{3} q_{1}^{4}+q_{3} q_{2}^{2}\right)-36 q_{1} q_{2} q_{3} \\
& +5\left(q_{1} q_{2}^{2} q_{3}^{2}+q_{2} q_{1}^{2} q_{3}^{2}+q_{3} q_{1}^{2} q_{2}^{2}\right), \\
\mathcal{F}_{3}^{1}= & -10+9\left(q_{1}+q_{2}+q_{3}\right)-8\left(q_{1} q_{2}+q_{1} q_{3}+q_{2} q_{3}\right)+7 q_{1} q_{2} q_{3}, \\
\mathcal{F}_{4}^{0}= & -192+286\left(q_{1}+q_{2}+q_{3}\right)-400\left(q_{1} q_{2}+q_{1} q_{3}+q_{2} q_{3}\right)-110\left(q_{1}^{2}+q_{2}^{2}+q_{3}^{2}\right) \\
& +135\left(q_{1} q_{2}^{2}+q_{1} q_{3}^{2}+q_{2} q_{1}^{2}+q_{2} q_{3}^{2}+q_{3} q_{1}^{4}+q_{3} q_{2}^{2}\right)+531 q_{1} q_{2} q_{3}-9\left(q_{1}^{3}+q_{2}^{3}+q_{3}^{2}\right) \\
& -8\left(q_{1} q_{2}^{3}+q_{1} q_{3}^{3}+q_{2} q_{1}^{3}+q_{2} q_{3}^{3}+q_{3} q_{1}^{3}+q_{3} q_{2}^{3}\right)-32\left(q_{1}^{2} q_{2}^{2}+q_{1}^{2} q_{3}^{2}+q_{2}^{2} q_{3}^{2}\right) \\
& -160\left(q_{1} q_{2} q_{3}^{2}+q_{1} q_{3} q_{2}^{2}+q_{2} q_{3} q_{1}^{2}\right)+35\left(q_{1} q_{2}^{2} q_{3}^{2}+q_{2} q_{3}^{3} q_{2}^{2}+q_{3} q_{1}^{2} q_{2}^{2}\right) \\
& +7\left(q_{1} q_{2} q_{3}^{3}+q_{1} q_{3} q_{2}^{3}+q_{2} q_{3} q_{1}^{3}\right)-6 q_{1}^{2} q_{2}^{2} q_{3}^{2},
\end{aligned}
$$


and finally,

$$
\begin{aligned}
\mathcal{F}_{4}^{1}= & 231-288\left(q_{1}+q_{2}+q_{3}\right)+344\left(q_{1} q_{2}+q_{1} q_{3}+q_{2} q_{3}\right)+68\left(q_{1}^{2}+q_{2}^{2}+q_{3}^{2}\right) \\
& -72\left(q_{1} q_{2}^{2}+q_{1} q_{3}^{2}+q_{2} q_{1}^{2}+q_{2} q_{3}^{2}+q_{3} q_{1}^{4}+q_{3} q_{2}^{2}\right)+396 q_{1} q_{2} q_{3} \\
& +9\left(q_{1}^{2} q_{2}^{2}+q_{1}^{2} q_{3}^{2}+q_{2}^{2} q_{3}^{2}\right)+74\left(q_{1} q_{2} q_{3}^{2}+q_{1} q_{3} q_{2}^{2}+q_{2} q_{3} q_{1}^{2}\right) \\
& -8\left(q_{1} q_{2}^{2} q_{3}^{2}+q_{2} q_{3}^{3} q_{2}^{2}+q_{3} q_{1}^{2} q_{2}^{2}\right) \\
\mathcal{F}_{4}^{2}= & -102+108\left(q_{1}+q_{2}+q_{3}\right)-112\left(q_{1} q_{2}+q_{1} q_{3}+q_{2} q_{3}\right)-12\left(q_{1}^{2}+q_{2}^{2}+q_{3}^{2}\right) \\
& +11\left(q_{1} q_{2}^{2}+q_{1} q_{3}^{2}+q_{2} q_{1}^{2}+q_{2} q_{3}^{2}+q_{3} q_{1}^{4}+q_{3} q_{2}^{2}\right)+114 q_{1} q_{2} q_{3} \\
& -10\left(q_{1} q_{2} q_{3}^{2}+q_{1} q_{3} q_{2}^{2}+q_{2} q_{3} q_{1}^{2}\right) \\
\mathcal{F}_{4}^{3}= & 15-14\left(q_{1}+q_{2}+q_{3}\right)+13\left(q_{1} q_{2}+q_{1} q_{3}+q_{2} q_{3}\right)-12 q_{1} q_{2} q_{3} .
\end{aligned}
$$

We can take the limit in which one of the $q_{i}$ 's, say $q_{3}$, goes to zero. The corresponding results for the $g=0$ amplitudes agree with those presented in [30] for the $\mathbb{B}_{2}$ local del Pezzo, after relabeling $t_{1,2} \rightarrow-t_{1,2}, r \rightarrow r+t_{1}+t_{2}$.

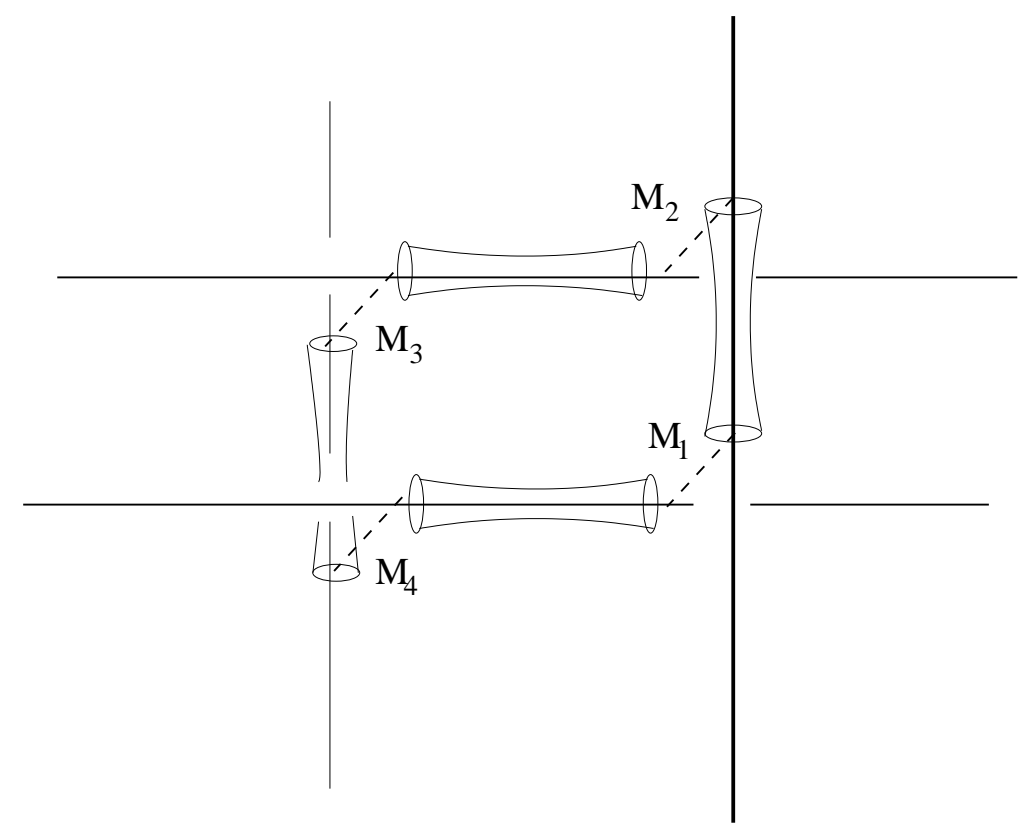

Fig. 14 The figure depicts four $\mathbf{S}^{3}$ 's connected with annuli.

\section{5. $\mathcal{O}(K) \rightarrow \mathbb{P}^{1} \times \mathbb{P}^{1}$}

We now consider the geometry that leads to local $\mathbb{P}^{1} \times \mathbb{P}^{1}$. In fig. 14 there are $N_{i}$ D-branes, $i=1, \ldots, 4$, wrapping a chain of four minimal spheres connecting two $(1,0)$ 
branes and two $(0,1)$ branes. For every pair of spheres "intersecting" over an $\mathbf{S}^{1}$ we get a bifundamental scalar field, so we have matter in representation $\left(N_{i}, \bar{N}_{i+1}\right)$, where $i=5$ corresponds to the first sphere again. The path integral of the A-model in this background can be written as:

$$
Z=\int \prod_{i=1}^{4} \mathcal{D} A_{i} e^{S_{\mathrm{CS}}\left(A_{i}\right)} \mathcal{O}\left(U_{1}, U_{2}\right) \mathcal{O}\left(U_{2}, U_{3}\right) \mathcal{O}\left(U_{3}, U_{4}\right) \mathcal{O}\left(U_{4}, U_{1}\right)
$$

There are two unknots on each three-sphere and the amplitude will depend on their linking numbers, in addition to framing. As before, we can use (4.5) to write this in a more transparent form

$$
\begin{aligned}
Z=\sum_{R_{1}, R_{2}, R_{3}, R_{4}}\left\langle V_{1} R_{1} \mid V_{4} R_{4}\right\rangle \mathrm{e}^{-\ell_{4} r_{4}}\left\langle V_{4} R_{4} \mid V_{3} R_{3}\right\rangle \mathrm{e}^{-\ell_{3} r_{3}} \\
\cdot\left\langle V_{3} R_{3} \mid V_{2} R_{2}\right\rangle \mathrm{e}^{-\ell_{2} r_{2}}\left\langle V_{2} R_{2} \mid V_{1} R_{1}\right\rangle \mathrm{e}^{-\ell_{1} r_{1}} .
\end{aligned}
$$

As in the previous case, the requisite diffeomorphism are determined by the geometry. From the figure, we have,

$$
V_{1}=S, \quad V_{2}=C, \quad V_{3}=S C, \quad V_{4}=\mathbf{1}
$$

This gives four $\mathbf{S}^{3}$ s, each of which has a Hopf link with linking number +1 and whose components have zero framing.

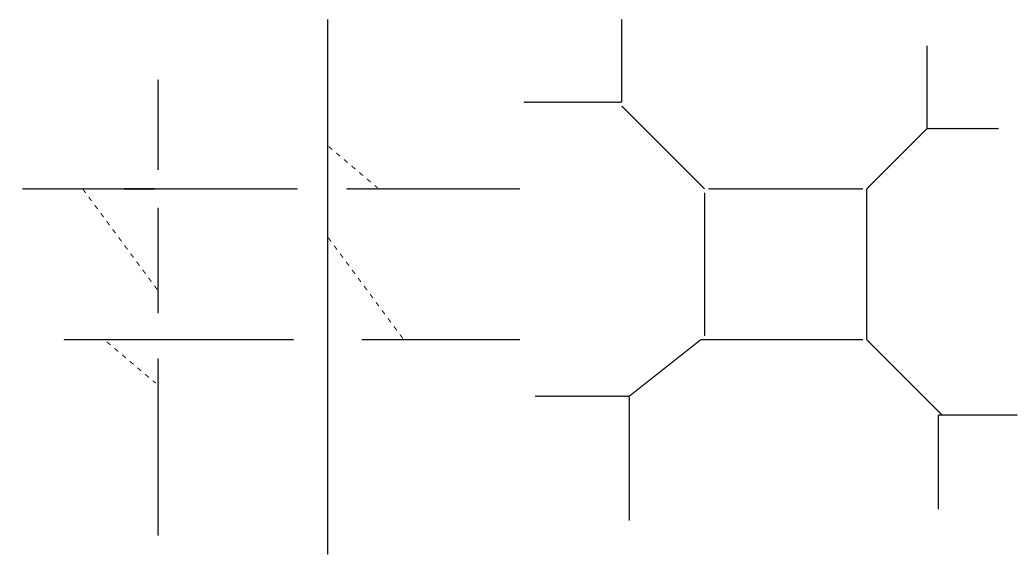

Fig. 15 The figure shows a geometric transition of four $\mathbf{S}^{3}$ in the previous figure. The dual geometry is related by four flops of the external $\mathbb{P}^{1}$ 's to a non-generic del Pezzo $\mathbb{B}_{5}$. 
The geometric transition is represented in fig. 15. The resulting dual closed string geometry contains a $\mathbb{P}^{1} \times \mathbb{P}^{1}$, together with four exceptional $\mathbb{P}^{1}$ 's. As in the previous case, we can take the limit $t_{i} \rightarrow \infty$ in order to extract the integer invariants of the local $\mathbb{P}^{1} \times \mathbb{P}^{1}$ geometry. The Chern-Simons computation that gives the invariants is very similar to the one we discussed in the previous section, so we won't give all the details. According to the geometric picture, we have four Chern-Simons theories with 't Hooft parameters $t_{1}, t_{2}, t_{3}$, and $t_{4}$, and with the same coupling constant $g_{s}$. Since all knots and links are identical, it is sufficient to label the vevs by indicating explicitly the corresponding 't Hooft parameter. The free energy at all genus for the topological closed string is then given by

$$
F=\log \left\{\sum_{R_{1}, R_{2}, R_{3}, R_{4}} \mathrm{e}^{-\ell_{1} r_{1}-\ell_{3} r_{2}-\ell_{2} s_{1}-\ell_{4} s_{2}} W_{R_{1}, R_{2}}\left(t_{1}\right) W_{R_{2}, R_{3}}\left(t_{2}\right) W_{R_{3}, R_{4}}\left(t_{3}\right) W_{R_{3}, R_{4}}\left(t_{4}\right)\right\} .
$$

Again, $r_{i}$ and $s_{i}$ are "bare" Kähler parameters that will lead to two "renormalized" Kähler parameter $r, s$. The relation between them can be obtained as in the previous case, and one easily finds:

$$
\begin{aligned}
& r=r_{1}-\frac{t_{1}+t_{4}}{2}=r_{2}-\frac{t_{2}+t_{3}}{2}, \\
& s=s_{1}-\frac{t_{1}+t_{2}}{2}=s_{2}-\frac{t_{3}+t_{4}}{2},
\end{aligned}
$$

and we have to rescale the Chern-Simons vevs as before,

$$
W_{R, R^{\prime}}\left(t_{i}\right) \rightarrow \lambda_{i}^{-\frac{\ell+\ell^{\prime}}{2}} W_{R, R^{\prime}}\left(t_{i}\right)
$$

Once we have done that, the free energy is given by:

$$
F=\log \left\{1+\sum_{\ell_{1}, \ell_{2}=1}^{\infty} a_{\ell_{1}, \ell_{2}}(q) \mathrm{e}^{-\ell_{1} r-\ell_{2} s}\right\}=\sum_{\ell_{1}, \ell_{2}=1}^{\infty} a_{\ell_{1}, \ell_{2}}^{(c)}(q) \mathrm{e}^{-\ell_{1} r-\ell_{2} s},
$$

and from here we can again extract the integral invariants $n_{\ell_{1}, \ell_{2}}^{g}$ by subtracting multicovering effects.

Let us present some explicit results at lower degree. For degrees $\left(\ell_{1}, \ell_{2}\right)=(1,0)$ and $(0,1)$, we find:

$$
\begin{aligned}
& a_{1,0}=\left(\lambda_{1} \lambda_{4}\right)^{-\frac{1}{2}} W_{\square}\left(t_{1}\right) W_{\square}\left(t_{4}\right)+\left(\lambda_{2} \lambda_{3}\right)^{-\frac{1}{2}} W_{\square}\left(t_{2}\right) W_{\square}\left(t_{3}\right), \\
& a_{0,1}=\left(\lambda_{1} \lambda_{2}\right)^{-\frac{1}{2}} W_{\square}\left(t_{1}\right) W_{\square}\left(t_{2}\right)+\left(\lambda_{3} \lambda_{4}\right)^{-\frac{1}{2}} W_{\square}\left(t_{3}\right) W_{\square}\left(t_{4}\right) .
\end{aligned}
$$


In general, the coefficients $a_{n, m}$ and $a_{m, n}$ are related by exchanging $t_{2} \leftrightarrow t_{4}$. By taking the limit $t_{i} \rightarrow \infty$, we find

$$
a_{1,0}=a_{0,1}=\frac{2}{\left(q^{-\frac{1}{2}}-q^{\frac{1}{2}}\right)^{2}},
$$

therefore

$$
n_{1,0}^{0}=n_{0,1}^{0}=-2,
$$

and the invariants for higher genus all vanish. This is indeed the right result [28] [30]. For $a_{1,1}^{(c)}$ we find:

$$
a_{1,1}^{(c)}=\lambda_{1}^{-1}\left(\lambda_{2} \lambda_{4}\right)^{-\frac{1}{2}} f_{(\square, \square)}\left(t_{1}\right) W_{\square}\left(t_{2}\right) W_{\square}\left(t_{4}\right)+\text { perms, }
$$

where perms stands for three terms that are obtained from the first one by permuting $t_{i} \rightarrow t_{i+1}$. Due to $(7.47)$, this has the structure of the degree $(1,1)$ term in a closed string free energy. After taking the limit $t_{i} \rightarrow \infty$, one finds

$$
a_{1,1}^{(c)}=\frac{4}{\left(q^{-\frac{1}{2}}-q^{\frac{1}{2}}\right)^{2}}
$$

therefore

$$
n_{1,1}^{0}=-4
$$

while the invariants for higher genera vanish. Again this is the right value for the invariant.

We can again easily implement the computation of these invariants. In the following tables we present most of the results up to total degree 10 and genus 8 (the non-trivial invariants for total degree 10 go all the way to genus 16 , which we have obtained, but have not included here for the economy of space):

\begin{tabular}{|rrrrrrrr|}
\hline$d_{2}$ & $d_{1}=0$ & 1 & 2 & 3 & 4 & 5 & 6 \\
\hline 0 & & -2 & 0 & 0 & 0 & 0 & 0 \\
\hline 1 & -2 & -4 & -6 & -8 & -10 & -12 & -14 \\
\hline 2 & 0 & -6 & -32 & -110 & -288 & -644 & -1280 \\
\hline 3 & 0 & -8 & -110 & -756 & -3556 & -13072 & -40338 \\
\hline 4 & 0 & -10 & -288 & -3556 & -27264 & -153324 & -690400 \\
\hline 5 & 0 & -12 & -644 & -13072 & -153324 & -1252040 & \\
\hline 6 & 0 & -14 & -1280 & -40338 & -690400 & & \\
\hline
\end{tabular}

Table 4: The integral invariants $n_{d}^{0}$ for the local $\mathbb{P}^{1} \times \mathbb{P}^{1}$ case. 


\begin{tabular}{|rrrrrr|}
\hline$d_{2}$ & $d_{1}=2$ & 3 & 4 & 5 & 6 \\
\hline 2 & 9 & 68 & 300 & 988 & 2698 \\
\hline 3 & 68 & 1016 & 7792 & 41376 & 172124 \\
\hline 4 & 300 & 7792 & 95313 & 760764 & 4552692 \\
\hline 5 & 988 & 41736 & 760764 & 8695048 & \\
\hline 6 & 2698 & 172124 & 4552692 & & \\
\hline
\end{tabular}

Table 5: The integral invariants $n_{d}^{1}$ for the local $\mathbb{P}^{1} \times \mathbb{P}^{1}$ case.

\begin{tabular}{|rrrrrr|}
\hline$d_{2}$ & $d_{1}=2$ & 3 & 4 & 5 & 6 \\
\hline 2 & 0 & -12 & -116 & -628 & -2488 \\
\hline 3 & -12 & -580 & -8042 & -64624 & -371980 \\
\hline 4 & -116 & -8042 & -167936 & -1964440 & -15913228 \\
\hline 5 & -628 & -64624 & -1964440 & -32242268 & \\
\hline 6 & -2488 & -371980 & -15913228 & & \\
\hline
\end{tabular}

Table 6: The integral invariants $n_{d}^{2}$ for the local $\mathbb{P}^{1} \times \mathbb{P}^{1}$ case.

\begin{tabular}{|rrrrrr|}
\hline$d_{2}$ & $d_{1}=2$ & 3 & 4 & 5 & 6 \\
\hline 2 & 0 & 0 & 15 & 176 & 1130 \\
\hline 3 & 0 & 156 & 4680 & 60840 & 501440 \\
\hline 4 & 15 & 4680 & 184056 & 3288688 & 36882969 \\
\hline 5 & 176 & 60840 & 3288688 & 80072160 & \\
\hline 6 & 1130 & 501440 & 36882969 & & \\
\hline
\end{tabular}

Table 7: The integral invariants $n_{d}^{3}$ for the local $\mathbb{P}^{1} \times \mathbb{P}^{1}$ case.

\begin{tabular}{|rrrrrr|}
\hline$d_{2}$ & $d_{1}=2$ & 3 & 4 & 5 & 6 \\
\hline 2 & 0 & 0 & 0 & -18 & -248 \\
\hline 3 & 0 & -16 & -1560 & -36048 & -450438 \\
\hline 4 & 0 & -1560 & -133464 & -3839632 & -61250176 \\
\hline 5 & -18 & -36048 & -3839632 & -144085372 & \\
\hline 6 & -248 & -450438 & -61250176 & & \\
\hline
\end{tabular}

Table 8: The integral invariants $n_{d}^{4}$ for the local $\mathbb{P}^{1} \times \mathbb{P}^{1}$ case. 


\begin{tabular}{|rrrrrr|}
\hline$d_{2}$ & $d_{1}=2$ & 3 & 4 & 5 & 6 \\
\hline 2 & 0 & 0 & 0 & 0 & 21 \\
\hline 3 & 0 & 0 & 276 & 13888 & 276144 \\
\hline 4 & 0 & 276 & 64973 & 3224340 & 75592238 \\
\hline 5 & 0 & 13888 & 3224340 & 195035824 & \\
\hline 6 & 21 & 276144 & 75592238 & & \\
\hline
\end{tabular}

Table 9: The integral invariants $n_{d}^{5}$ for the local $\mathbb{P}^{1} \times \mathbb{P}^{1}$ case.

\begin{tabular}{|rrrrr|}
\hline$d_{2}$ & $d_{1}=3$ & 4 & 5 & 6 \\
\hline 3 & 0 & -20 & -3260 & -115744 \\
\hline 4 & -20 & -20936 & -1969710 & -70665312 \\
\hline 5 & -3260 & -1969710 & -202598268 & \\
\hline 6 & -115744 & -70665312 & & \\
\hline
\end{tabular}

Table 10: The integral invariants $n_{d}^{6}$ for the local $\mathbb{P}^{1} \times \mathbb{P}^{1}$ case.

\begin{tabular}{|rrrrr|}
\hline$d_{2}$ & $d_{1}=3$ & 4 & 5 & 6 \\
\hline 3 & 0 & 0 & 428 & 32568 \\
\hline 4 & 0 & 4266 & 873972 & 50501308 \\
\hline 5 & 428 & 873972 & 163185964 & \\
\hline 6 & 32568 & 50501308 & & \\
\hline
\end{tabular}

Table 11: The integral invariants $n_{d}^{7}$ for the local $\mathbb{P}^{1} \times \mathbb{P}^{1}$ case.

\begin{tabular}{|rrrrr|}
\hline$d_{2}$ & $d_{1}=3$ & 4 & 5 & 6 \\
\hline 3 & 0 & 0 & -24 & -5872 \\
\hline 4 & 0 & -496 & -277880 & -27655024 \\
\hline 5 & -24 & -277880 & -102321184 & \\
\hline 6 & -5872 & -27655024 & & \\
\hline
\end{tabular}

Table 12: The integral invariants $n_{d}^{8}$ for the local $\mathbb{P}^{1} \times \mathbb{P}^{1}$ case.

These results are in full agreement with the ones presented in [28] [30] 31]. Again, we can verify many of these numbers with the geometric formulae of [31]. For a given bidegree $(a, b), n_{(a, b)}^{g}$ vanishes for $g>(a-1)(b-1)$, which is indeed the arithmetic genus of a curve of bidegree $(a, b)$ in $\mathbb{P}^{1} \times \mathbb{P}^{1}$. One finds,

$$
n_{(a, b)}^{(a-1)(b-1)}=-(-1)^{(a+1)(b+1)}(a+1)(b+1)
$$


which reproduces the corresponding results listed in the tables above. For curves with one node, one finds:

$$
n_{(a, b)}^{(a-1)(b-1)-1}=2(-1)^{(a+1)(b+1)}\left(a+b+a b-a^{2}-b^{2}+a^{2} b^{2}\right)
$$

again in full agreement with the tables. For curves with two nodes (extending the derivation in [31]) we have:

$$
\begin{aligned}
n_{(a, b)}^{(a-1)(b-1)-2}=- & (-1)^{(a+1)(b+1)}\left(-14+9(a+b)-3 a b-3\left(a^{2}+b^{2}\right)+3 a^{2} b^{2}\right. \\
& \left.+2\left(a^{3}+b^{3}+a^{2} b+b^{2} a\right)-2\left(a^{3} b+b^{3} a\right)-2\left(a^{3} b^{2}+b^{3} a^{2}\right)+2 a^{3} b^{3}\right),
\end{aligned}
$$

which reproduces for example $n_{(3,3)}^{2}=-580, n_{(3,6)}^{8}=-5872$ and $n_{(4,4)}^{7}=4266$. For the invariants corresponding to bidegrees $(2, n)$, where $3 \leq n \leq 6$, and curves with two nodes, one has to introduce corrections associated to reducible curves. For example, for bidegrees $(2,6),(7.74)$ gives the value 1166 , but there are reducible curves of type $(5,2) \cup(1,0)$ with two nodes. Since $n_{(1,0)}^{0}=-2$, and $n_{(5,2)}^{4}=-18$, the subtraction scheme proposed in [31] gives $n_{(2,6)}^{3}=1166-(-2)(-18)=1130$, in agreement with the result of table 7 .

\subsection{Refined Integral Invariants}

The integral invariants $n_{d}^{g}$ defined in [57 denote the (net) number of wrapped M2 branes in $4+1$ dimensional effective theory, obtained by compactification of M-theory on the corresponding Calabi-Yau, where $d \in H_{2}(X)$ denotes the class the M2 brane is wrapped and $g$ denotes a basis for the $S U(2)_{L}$ rotation subgroup of $S O(4)$ (see [57] for details). If the Calabi-Yau space has global symmetries, then these states also form representations of this group. Compact Calabi-Yau manifolds do not admit global symmetries, so this does not arise in that context. However for local toric 3-folds there always are extra global symmetries and one can ask how the $n_{d}^{g}$ decompose in representations of this symmetry algebra. Thus it is natural to ask whether we can use our techniques to also compute these refined invariants.

For example, consider the linear sigma model describing $\mathcal{O}(-3) \rightarrow \mathbb{P}^{2}$, which contains three matter fields of charge +1 . In this case there are two extra $U(1)$ global symmetries, which for some metric in $\mathbb{P}^{2}$ could give rise to the Cartan of $S U(3)$. This can be implemented in terms of the toric diagram, by assigning different sizes to the different edges. In the local $\mathbb{P}^{2}$ case, we should assign different sizes to the triangle describing the base of the 
$\mathbb{P}^{2}$, i.e. we should introduce three Kähler parameters instead of one. Notice that this is perfectly natural from the point of view of the Chern-Simons description, because in the limit where we took the $N_{i} \rightarrow \infty$ we had to tune $r$ 's. Nothing prevents us from tuning the three edges to different values by considering a suitable limit.

Let us consider the computation of the refined integral invariants in some detail, in the case of local $\mathbb{P}^{2}$. We have to introduce three parameters associated to the three different edges, and we will denote them by $r_{i}^{\prime}$, with $i=1,2,3$, where we view $e^{-r_{i}^{\prime}}$ as forming a Cartan torus of $U(3)$. Notice that, if we write $U(3)=U(1) \times S U(3)$, the overall $U(1)$ quantum number is precisely the degree $d$. We then have to further decompose the spectrum with respect to the $S U(3)$. This goes as follows: due to the underlying symmetry, the closed string free energy will be now of the form:

$$
\sum_{m=1}^{\infty} \sum_{g, d} n_{d}^{g}\left(x_{1}^{m}, x_{2}^{m}, x_{3}^{m}\right) \frac{1}{m}\left(2 \sin \frac{m g_{s}}{2}\right)^{2 g-2}
$$

where $x_{i}=\mathrm{e}^{-r_{i}^{\prime}}, i=1,2,3$, and $n_{d}^{g}\left(x_{1}, x_{2}, x_{3}\right)$ is now a symmetric polynomial of degree $d$ in the $x_{i}$, with integer coefficients. Therefore, we can expand it in terms of Schur polynomials $s_{R}$ in three variables and of degree $d$, which are labeled by representations $R$ of $S U(3)$ with $d$ boxes. We then write:

$$
n_{d}^{g}\left(x_{1}, x_{2}, x_{3}\right)=\sum_{R} n_{d, R}^{g} s_{R}\left(x_{1}, x_{2}, x_{3}\right)
$$

where the sum is over representations of $S U(3)$ with $d$ boxes, and $n_{d, R}^{g}$ denote the number of M2 branes of degree $d$ with $S U(2)_{L}$ representation $g$ and transforming as representation $R$ of the $S U(3)$ global symmetry. These are the refined invariants of the local $\mathbb{P}^{2}$. Notice that, if we put $x_{1}=x_{2}=x_{3}=1$ in (7.76), we recover the usual integer invariants, therefore one has

$$
n_{d}^{g}=\sum_{R}(\operatorname{dim} R) n_{d, R}^{g}
$$

The computation of the refined invariants can be easily done in the Chern-Simons setting, by taking the renormalized sizes of the annuli to be different. The renormalized sizes will give in this way the parameters $r_{i}^{\prime}$ appearing in the closed string side, in other words:

$$
r_{1}^{\prime}=r-\frac{t_{1}+t_{2}}{2}
$$


and so on. The refined invariants for the first few degrees can be easily computed. At degree one we find,

$$
n_{1}^{0}\left(x_{1}, x_{2}, x_{3}\right)=x_{1}+x_{2}+x_{3}
$$

therefore $n_{1, \square}^{0}=1$. At degree two, one has:

$$
n_{2, \square}^{0}=-1, \quad n_{2, \Theta}^{0}=0 .
$$

At degree three, we find:

$$
\begin{array}{lll}
n_{3, \text { एा }}^{0}=2 & n_{3, \Xi}^{0}=1, & n_{3, \text {, }}^{0}=-1, \\
n_{3, \text { ए巴 }}^{1}=-1 & n_{3, \Xi}^{1}=0, & n_{3, \text {, }}^{0}=0,
\end{array}
$$

We finally list the results for degree four:

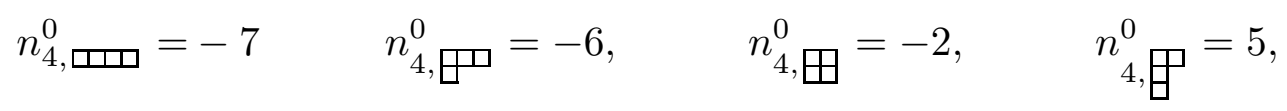

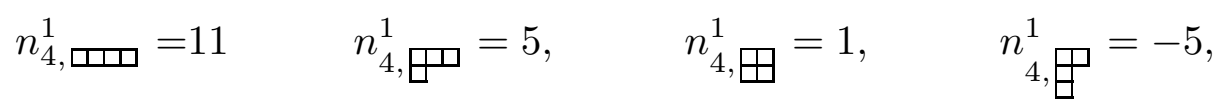

$$
\begin{aligned}
& n_{4, \text { 口س }}^{2}=-6 \quad n_{4, \text { Ш }}^{2}=-1, \quad n_{4, \text { 田 }}^{2}=0, \quad n_{4, \boxplus}^{2}=-1 ，
\end{aligned}
$$

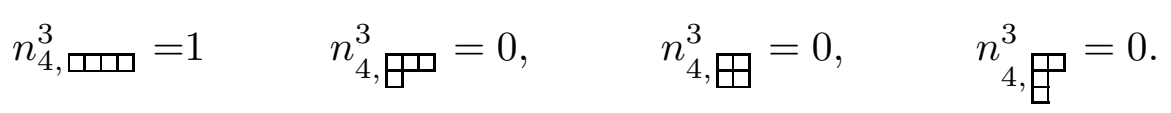

For $\mathbb{P}^{1} \times \mathbb{P}^{1}$ one can similarly decompose the invariants with respect to the $S U(2) \times$ $S U(2)$ global symmetry of the model. Note that from a mathematical point of view, these refined integer invariants should be related to the equivariant Gromov-Witten invariants associated to the group action on the manifold, as was studied in the Fano case in [61]. Moreover, one could use the techniques of 61 to obtain the mirror of these deformations for the toric Calabi-Yau manifolds we have discussed and check the results obtained here against the predictions of mirror symmetry (at least for genus 0).

\section{Embedding in Superstrings}

It is natural to ask what kind of dualities these geometric transitions lead to, once we embed them in superstrings, as was done in [11] for the original Chern-Simons duality [1].

Embedding these dualities for topological strings in type IIA strings is easily done by replacing the branes with D6 branes wrapping $\mathbf{S}^{3}$ 's and filling 4 dimensional spacetime. 
Thus we end up, at low energy with a system involving $\mathcal{N}=1 U\left(N_{i}\right)$ gauge symmetry. Moreover for each annulus contribution we end up with a bifundamental matter "hypermultiplet" in the superstring context. Of course this is only the low energy limit of the brane system. The high energy aspects of this theory differ from that of pure Yang-Mills. This can be deduced by considering the superpotential for this theory, as was done in [11]. In the IR the gaugino condensation will take place where the $\mathbf{S}^{3}$ 's are replaced by blown up $\mathbf{S}^{2}$ 's with RR fluxes through them. There is no RR flux through $\mathbf{S}^{2}$ 's which come from matter bifundamentals. In the applications we have looked at, we have also considered the interesting limit where the sizes of blown up $\mathbf{S}^{2}$ 's go to infinity, while keeping the effective masses of the bifundamental fields finite. This was, for example, how we got the full answer for $\mathbb{P}^{2}$ in topological strings. In the gauge theory setup the size of the blown up $\mathbf{S}^{2}$ 's correspond to the size of the gaugino condensate getting large, which can be adjusted by increasing the corresponding gauge coupling. Note that in this limit we will have no RR flux left in the type IIA superstring theory. Since we have fixed total RR flux through the $\mathbf{S}^{2}$ 's which get infinitely large, in the limit we are considering the flux per unit volume goes to zero. Moreover, the finite $\mathbf{S}^{2}$ 's in this limit correspond to where the bifundamental matter came from, and there is no flux through them. Thus we end up with a novel large $N$ duality, were the bifundamental matter structure dictates the geometry of the dual and this geometry has no RR flux in it.

The statement of the above dualities correspond to gauge theories with all the string interactions on them. One would naturally ask if there are any large $N$ dualities along these lines for pure gauge theories. For this purpose it is convenient to go to the type IIB mirror setup.

To illustrate the idea let us first consider a simple example. Consider the $\mathcal{N}=2$, $U(2 N)$ gauge theory deformed by the addition of superpotential

$$
W=g \operatorname{tr}\left[\frac{1}{3} \Phi^{3}-m^{2} \Phi\right]
$$

where $\Phi$ is the adjoint field. There are two classical values for the eigenvalues of $\Phi$, namely $\Phi= \pm m$. Let us choose $N$ eigenvalues of $\Phi$ to be at $+m$ and $N$ to be at $-N$. Then the large $N$ dual of this system in type IIB is proposed in 177 (and further elaborated recently in [62]) to be given by propagation in the non-compact Calabi-Yau given by the hypersurface in $\mathbb{C}^{4}$ :

$$
u v+y^{2}+g^{2}\left(x^{2}-m^{2}\right)^{2}+g^{2} \Lambda^{4}=0
$$


where $\Lambda$ is related to the scale of the original $\mathcal{N}=2$ theory. Note that for small $\Lambda$ we have two conifold points centered near $x=m$ and $x=-m$. In this dual gravitational geometry, there is RR flux of $N$ units through each of the corresponding $\mathbf{S}^{3}$ 's. However there is no RR flux through the compact $\mathbf{S}^{3}$ which runs between these two $\mathbf{S}^{3}$ 's (and intersects both at 1 point). It is convenient to rewrite the above geometry as

$$
u v+y^{2}+g^{2} P(x)=0
$$

where

$$
P(x)=\left(x^{2}-M^{2}\right)\left(x^{2}-a^{2}\right)
$$

and we identify

$$
\begin{aligned}
& m^{2}=\frac{1}{2}\left(M^{2}+a^{2}\right) \\
& \Lambda^{4}=\frac{-\left(M^{2}-a^{2}\right)^{2}}{4}
\end{aligned}
$$

In this parameterization the two $\mathbf{S}^{3}$ 's with $\mathrm{RR}$ flux project in the x-plane to the intervals $-M \leq x \leq-a$ and $a \leq x \leq M$. In particular there is no flux through the $\mathbf{S}^{3}$ which projects to the interval $-a \leq x \leq a$. We consider the situation where $(M / a)>>1$. In this limit the two $\mathbf{S}^{3}$ 's have become big. In particular as $M \rightarrow \infty$, keeping $a$ and $\alpha=-g^{2} M^{2}$ fixed the geometry reduces to

$$
u v+y^{2}+\alpha\left(x^{2}-a^{2}\right)=0
$$

which is the ordinary conifold. Moreover, in this limit the RR fields per unit volume go to zero everywhere. Thus we have found a gauge theory/gravity duality where the geometry is free of RR flux. To be precise we have to note that we need to complete the duality by going farther in the UV of gauge system, which forces a cascade structure [19] generalizing the construction of [16] to the case at hand. Namely, we will end up with a $U(2 N+M) \times U(M)$ gauge system, with two bifundamental hypermultiplets, as $M \rightarrow \infty$. Moreover we have superpotentials $W_{1}\left(\Phi_{1}\right)$ and $W_{2}\left(\Phi_{2}\right)$ which have the same functional form as the superpotential $W(\Phi)$ discussed before, namely $W_{1}=-W_{2}=W$, where the coefficients of $W$ are carefully tuned, as discussed above. Thus we have a proposal for a gauge dual description of the standard conifold with no flux through it.

Clearly this example can be generalized. In fact a large class of local Calabi-Yau threefolds were constructed in [18] as duals to gauge systems, which were analyzed in [19]. 
Applying a similar kind of reasoning as the above example we end up describing a rather large class of local threefold without fluxes, as duals to some limits of $\mathcal{N}=2$ gauge systems

deformed to $\mathcal{N}=1$ by superpotential terms. It would be very interesting to study the physical implications of these dualities.

\section{Acknowledgements}

We would like to thank E. Diaconescu, A. Grassi, S. Katz and P. Ramadevi for valuable discussions.

This research is supported in part by NSF grants PHY-9802709 and DMS-0074329. 


\section{References}

[1] R. Gopakumar and C. Vafa, "On the gauge theory/geometry correspondence," hepth/9811131, Adv. Theor. Math. Phys. 3 (1999) 1415.

[2] H. Ooguri and C. Vafa, "Knot invariants and topological strings," hep-th/9912123, Nucl. Phys. B 577 (2000) 419.

[3] J.M.F. Labastida and M. Mariño, "Polynomial invariants for torus knots and topological strings," hep-th/0004196, Commun. Math. Phys. 217 (2001) 423.

[4] P. Ramadevi and T. Sarkar, "On link invariants and topological string amplitudes," hep-th/0009188, Nucl. Phys. B 600 (2001) 487.

[5] J.M.F. Labastida, M. Mariño and C. Vafa, "Knots, links and branes at large $N$," hep-th/0010102, JHEP 0011 (2000) 007.

[6] J. M. Labastida and M. Mariño, "A new point of view in the theory of knot and link invariants," math.QA/0104180, J. Knot Theory Ramifications 11 (2002) 173.

[7] M. Aganagic, A. Klemm and C. Vafa, "Disk instantons, mirror symmetry and the duality web," hep-th/0105045, Z. Naturforsch. A 57 (2002) 1.

[8] M. Mariño and C. Vafa, "Framed knots at large $N$," hep-th/0108064.

[9] S. Sinha and C. Vafa, "SO and Sp Chern-Simons at large $N$," hep-th/0012136.

[10] B. Acharya, M. Aganagic, K. Hori and C. Vafa, "Orientifolds, mirror symmetry and superpotentials," hep-th/0202208.

[11] C. Vafa, "Superstrings and topological strings at large $N$," hep-th/0008142, J. Math. Phys. 42 (2001) 2798.

[12] M. Atiyah, J. M. Maldacena and C. Vafa, "An M-theory flop as a large N duality," hep-th/0011256, J. Math. Phys. 42 (2001) 3209.

[13] B. Acharya, "On realising $\mathcal{N}=1$ super Yang-Mills in M theory," hep-th/0011089.

[14] M. Aganagic and C. Vafa, "Mirror symmetry and a G(2) flop," hep-th/0105225.

[15] M. Atiyah and E. Witten, "M-theory dynamics on a manifold of G(2) holonomy," hep-th/0107177.

[16] I. R. Klebanov and M. J. Strassler, "Supergravity and a confining gauge theory: Duality cascades and chiSB-resolution of naked singularities," hep-th/0007191, JHEP 0008 (2000) 052.

[17] F. Cachazo, K. A. Intriligator and C. Vafa, "A large $N$ duality via a geometric transition," hep-th/0103067, Nucl. Phys. B 603 (2001) 3.

[18] F. Cachazo, S. Katz and C. Vafa, "Geometric transitions and N = 1 quiver theories," hep-th/0108120.

[19] F. Cachazo, B. Fiol, K. A. Intriligator, S. Katz and C. Vafa, "A geometric unification of dualities," hep-th/0110028, Nucl. Phys. B 628 (2002) 3.

[20] J. D. Edelstein, K. Oh and R. Tatar, "Orientifold, geometric transition and large N duality for SO/Sp gauge theories," hep-th/0104037, JHEP 0105 (2001) 009. 
[21] K. Dasgupta, K. Oh and R. Tatar, "Geometric transition, large N dualities and MQCD dynamics," hep-th/0105066, Nucl. Phys. B 610 (2001) 331; "Open/closed string dualities and Seiberg duality from geometric transitions in M-theory," hep-th/0106040; "Geometric transition versus cascading solution," hep-th/0110050, JHEP 0201 (2002) 031.

[22] H. Fuji and Y. Ookouchi, "Confining phase superpotentials for SO/Sp gauge theories via geometric transition," hep-th/0205301.

[23] A. Giveon, A. Kehagias and H. Partouche, "Geometric transitions, brane dynamics and gauge theories," hep-th/0110115, JHEP 0112 (2001) 021.

[24] H. Ooguri and C. Vafa, "Worldsheet derivation of a large $N$ duality," hep-th/0205297.

[25] M. Aganagic and C. Vafa, "G(2) manifolds, mirror symmetry and geometric engineering," hep-th/0110171.

[26] E. Witten, "Chern-Simons gauge theory as a string theory," hep-th/9207094, in The Floer memorial volume, H. Hofer, C.H. Taubes, A. Weinstein and E. Zehner, eds., Birkhäuser 1995, p. 637.

[27] E. Diaconescu, B. Florea and A. Grassi, "Geometric transitions and open string instantons," hep-th/0205234.

[28] S. Katz, A. Klemm and C. Vafa, "Geometric engineering of quantum field theories," hep-th/9609239, Nucl. Phys. B 497 (1997) 173.

[29] A. Klemm and E. Zaslow, "Local mirror symmetry at higher genus," hep-th/9906046, in Winter School on Mirror Symmetry, Vector bundles and Lagrangian Submanifolds, p. 183, American Mathematical Society 2001.

[30] T. M. Chiang, A. Klemm, S. T. Yau and E. Zaslow, "Local mirror symmetry: Calculations and interpretations," hep-th/9903053, Adv. Theor. Math. Phys. 3 (1999) 495.

[31] S. Katz, A. Klemm and C. Vafa, "M-theory, topological strings and spinning black holes," hep-th/9910181, Adv. Theor. Math. Phys. 3 (1999) 1445.

[32] D.-E. Diaconescu, B. Florea and A. Grassi, "Geometric transitions, del Pezzo surfaces and open string instantons," to appear.

[33] O. Aharony and A. Hanany, "Branes, superpotentials and superconformal fixed points," hep-th/9704170, Nucl. Phys. B 504 (1997) 239. O. Aharony, A. Hanany and B. Kol, "Webs of $(\mathrm{p}, \mathrm{q})$ 5-branes, five dimensional field theories and grid diagrams," hep-th/9710116, JHEP 9801 (1998) 002.

[34] N. C. Leung and C. Vafa, "Branes and toric geometry," hep-th/9711013, Adv. Theor. Math. Phys. 2 (1998) 91.

[35] M. Aganagic, A. Karch, D. Lust and A. Miemiec, "Mirror symmetries for brane configurations and branes at singularities," hep-th/9903093, Nucl. Phys. B 569 (2000) 277. 
[36] E. Witten, "Quantum field theory and the Jones polynomial," Commun. Math. Phys. 121 (1989) 351.

[37] E. Witten, "Noncommutative geometry and string field theory," Nucl. Phys. B 268 (1986) 253.

[38] M. Bershadsky, C. Vafa and V. Sadov, "D-strings on D-manifolds," hep-th/9510225, Nucl. Phys. B 463 (1996) 398.

[39] M. Bershadsky, S. Cecotti, H. Ooguri and C. Vafa, "Kodaira-Spencer theory of gravity and exact results for quantum string amplitudes," hep-th/9309140, Commun. Math. Phys. 165 (1994) 311.

[40] E. Guadagnini, M. Martellini and M. Mintchev, "Wilson lines in Chern-Simons theory and link invariants," Nucl. Phys. B 330 (1990) 575.

[41] S. Katz and M. Liu, "Enumerative geometry of stable maps with Lagrangian boundary conditions and multiple covers of the disc," math.AG/0103074, Adv. Theor. Math. Phys. 5 (2002) 1.

[42] T. Graber and E. Zaslow, "Open-string Gromov-Witten invariants: calculations and a mirror 'theorem'," hep-th/0109075.

[43] E. Witten, "On quantum gauge theories in two-dimensions," Commun. Math. Phys. 141 (1991) 153.

[44] S. Cordes, G. W. Moore and S. Ramgoolam, "Lectures on 2-d Yang-Mills theory, equivariant cohomology and topological field theories," hep-th/9411210, Nucl. Phys. Proc. Suppl. 41 (1995) 184.

[45] E. Verlinde, "Fusion rules and modular transformations in 2-D conformal field theory," Nucl. Phys. B 300 (1988) 360.

[46] E. Witten, "Phases of $\mathcal{N}=2$ theories in two dimensions," hep-th/9301042, Nucl. Phys. B 403 (1993) 159.

[47] S. Katz, P. Mayr and C. Vafa, "Mirror symmetry and exact solution of 4D $\mathcal{N}=2$ gauge theories. I," hep-th/9706110, Adv. Theor. Math. Phys. 1 (1998) 53.

[48] M. Kontsevich, "Enumeration of rational curves via torus actions," hep-th/9405035, in The moduli space of curves, p. 335, Birkhäuser, 1995.

[49] T. Graber and R. Pandharipande, "Localization of virtual classes," alg-geom/9708001, Invent. Math. 135 (1999) 487.

[50] M. Kontsevich, "Intersection theory on the moduli space of curves and the matrix Airy function," Commun. Math. Phys. 147 (1992) 1.

[51] C. Faber, "Algorithms for computing intersection numbers of curves, with an application to the class of the locus of Jacobians," alg-geom/9706006, in New trends in algebraic geometry, Cambridge Univ. Press, 1999.

[52] J.M.F. Labastida, P. M. Llatas and A. V. Ramallo, "Knot operators in Chern-Simons gauge theory," Nucl. Phys. B 348 (1991) 651. 
[53] J.M. Isidro, J.M.F. Labastida and A.V. Ramallo, "Polynomials for torus links from Chern-Simons gauge theory," hep-th/9210124, Nucl. Phys. B 398 (1993) 187.

[54] H.R. Morton and S.G. Lukac, "The HOMFLY polynomial of the decorated Hopf link," math.GT/0108011.

[55] S.G. Lukac, "HOMFLY skeins and the Hopf link," Ph.D. Thesis, June 2001, in http://www.liv.ac.uk/ su14/knotgroup.htm

[56] I.G. Macdonald, Symmetric functions and Hall polynomials, 2nd edition, Oxford University Press, 1995.

[57] R. Gopakumar and C. Vafa, "M-theory and topological strings, II," hep-th/9812127.

[58] R. K. Kaul, "Chern-Simons theory, knot invariants, vertex models and three-manifold invariants," hep-th/9804122, in Frontiers of field theory, quantum gravity and strings, p. 45, Nova Science, 1999.

[59] R.K. Kaul and P. Ramadevi, "Three-manifold invariants from Chern-Simons field theory with arbitrary semi-simple gauge groups," hep-th/0005096, Commun. Math. Phys. 217 (2001) 295.

[60] J. Bryan and R. Pandharipande, "BPS states of curves in Calabi-Yau threefolds," math.AG/0009025, Geom. Topolo. 5 (2001) 287.

[61] K. Hori and C. Vafa, "Mirror symmetry," hep-th/0002222.

[62] F. Cachazo and C. Vafa, "N $\mathcal{N}=1$ and $\mathcal{N}=2$ geometry from fluxes," hep-th/0206017. 